\title{
12. ORGANIC MATTER AND PALEOCHEMISTRY OF CRETACEOUS SEDIMENTS FROM THE ARGO AND GASCOYNE ABYSSAL PLAINS, NORTHEASTERN INDIAN OCEAN ${ }^{1}$
}

\author{
David T. Heggie ${ }^{2}$
}

\begin{abstract}
The accumulation of organic matter, ferrous and pyrite iron, and the ratios of organic carbon/total sulfur and organic carbon/total phosphorus in the Lower Cretaceous sediments from the Argo and Gascoyne abyssal plains have been used as indicators of both the source and reactivity of organic matter in the sediments and the depositional environment.

Total sulfur, used as an indicator of pyrite sulfur, is more abundant in sediments from the Gascoyne Abyssal Plain than in those from the Argo Abyssal Plain. Sulfur positively correlates with TOC at both sites (although poorly at the Argo Abyssal Plain site, R $=0.48$ ), with an extension of the line of best-fit through the origin, indicating that pyrite (TOC $<2 \mathrm{wt} \%$ ) is diagenetic and deposited from normal marine conditions. The average ratio of $\mathrm{C} / \mathrm{S}$ for samples of TOC $<2 \mathrm{wt} \%$ is 5.4 at Argo Abyssal Plain (compared to the modern normal marine value of 2.8) indicating deposition of organic matter probably of mixed terrestrial and oxidized marine sources that is unreactive to the sulfate-reducing bacteria. One sample from the Aptian sediments is rich in TOC (5.1 wt\%) and has a C/S ratio of 0.5. The average C/S ratio in Gascoyne Abyssal Plain sediments is $0.8(R=0.97)$, which indicates the formation of abundant pyrite in addition to burial and preservation of relatively fresh organic matter that is reactive to the sulfate-reducing bacteria. Organic carbon to phosphorus ratios $(\mathrm{C} / \mathrm{P})$ in the sediments indicate preferential remobilization of organic carbon over phosphorus with increasing water depth.

Estimates of the degree of pyritization (DOP) increase with increasing TOC at both sites, indicating iron is not limiting and pyrite is formed diagenetically. The one sample with a TOC content of $5.1 \mathrm{wt} \%$, from the Argo Abyssal Plain near the Barremian-Aptian boundary, is composed mostly of framboidal pyrite, finely laminated and not bioturbated, and hence may have been deposited during a brief period of anoxia in the overlying waters.
\end{abstract}

\section{INTRODUCTION}

Drilling data from Site 765 in the Argo Abyssal Plain (presentday water depth, $5725 \mathrm{~m}$; Fig. 1) and Site 766 at the foot of the Exmouth Plateau escarpment in the Gascoyne Abyssal Plain (present-day water depth, $3997.5 \mathrm{~m}$; Fig.1) indicate sediment types and total organic carbon (TOC) contents in the Cretaceous sections (Ludden, Gradstein, et al., 1990) that reflect different depositional environments in this part of the incipient northeastern Indian Ocean.

On the Argo Abyssal Plain, $\sim 500 \mathrm{~m}$ of zeolitic and siliceous clays, calcareous ooze, and turbidites overlie condensed Paleogene and Upper Cretaceous sections and $\sim 400 \mathrm{~m}$ of Lower Cretaceous sediments. Calcareous and siliciclastic turbidites and radiolarites are evident in these Lower Cretaceous sediments, although the Berriasian through Barremian sections (805-971 m below seafloor [mbsf]) and the Aptian/Albian sections (591-805 mbsf) are dominated by alternating bands of various thicknesses, over scales of centimeters to meters, of red, brown, green, dark-gray, and black claystones with various degrees of bioturbation (von Rad et al., 1989; Ludden, Gradstein, et al., 1990).

On the Gascoyne Abyssal Plain, $\sim 100 \mathrm{~m}$ of Cenozoic pelagic calcareous and siliceous ooze overlie $\sim 370 \mathrm{~m}$ of Cretaceous sediments, of which $>300 \mathrm{~m}$ were deposited during the Early Cretaceous. These Lower Cretaceous sediments from the Gascoyne Abyssal Plain are dominated by mixtures of greenish-gray/black siltstones and sandstones (Valanginian/Barremian), while the Aptian through Albian sediments are tan to light-green nannofossil

\footnotetext{
${ }^{1}$ Gradstein, F. M., Ludden, J. N., et al., 1992. Proc, ODP. Sci. Results, 123 College Station, TX (Ocean Drilling Program).

${ }^{2}$ Bureau of Mineral Resources, Division of Marine Geosciences and Petroleum Geology, P.O. Box 378, Canberra, ACT, 2601, Australia.
}

chalks which are clayey, silicified, and zeolitic (von Rad et al., 1989; Ludden, Gradstein, et al., 1990).

The objectives of this research were (1) to compare and contrast the Early Cretaceous depositional environments at the two sites, (2) to comment upon the reactivity and source(s) of organic matter, and (3) to comment upon oxic/anoxic conditions in the sediments and overlying waters. This study was prompted, in part, by (1) the shipboard observations of the patterns of color variations in the sediments at Site 765 (black, dark-green/gray-green, and red claystones) and the probability that these indicated different abundances of diagenetic iron minerals as a result of variable TOC fluxes, source(s), and reactivity of the organic matter, (2) the obvious contrast between sediments of comparable ages at the two sites, and (3) the single occurrence of a thin $(1 \mathrm{~cm})$ section of high TOC content $(5.1 \mathrm{wt} \%)$ in the Aptian sediments of the Argo Abyssal Plain, possibly representing the record of an isolated anoxic water column event.

During this study, I examined estimates of the abundances of some diagenetic products of organic matter degradation that provide clues to the depositional environment, e.g., sediment TOC sources and the reactivity of the organic matter and bottom water oxygen conditions. The following estimates are reported on here: (1) total organic carbon (TOC) and total sulfur (an estimate of pyrite sulfur) contents; (2) organic carbon/total sulfur ratios (hereafter shown as $\mathrm{C} / \mathrm{S}$ ); (3) limited iron speciation (ferrous iron and pyrite iron abundances); (4) estimates of DOP (degree of pyritization); (5) total phosphorus contents; and (6) organic carbon/ total phosphorus ratios (hereafter reported as $\mathrm{C} / \mathrm{P}$ ) in the sediments.

\section{METHODS}

Core samples were analyzed for TOC by Rock-Eval pyrolysis and calcium carbonate by coulometry (Emeis and Kvenvolden, 1986). These data are summarized in Ludden, Gradstein, et al. 


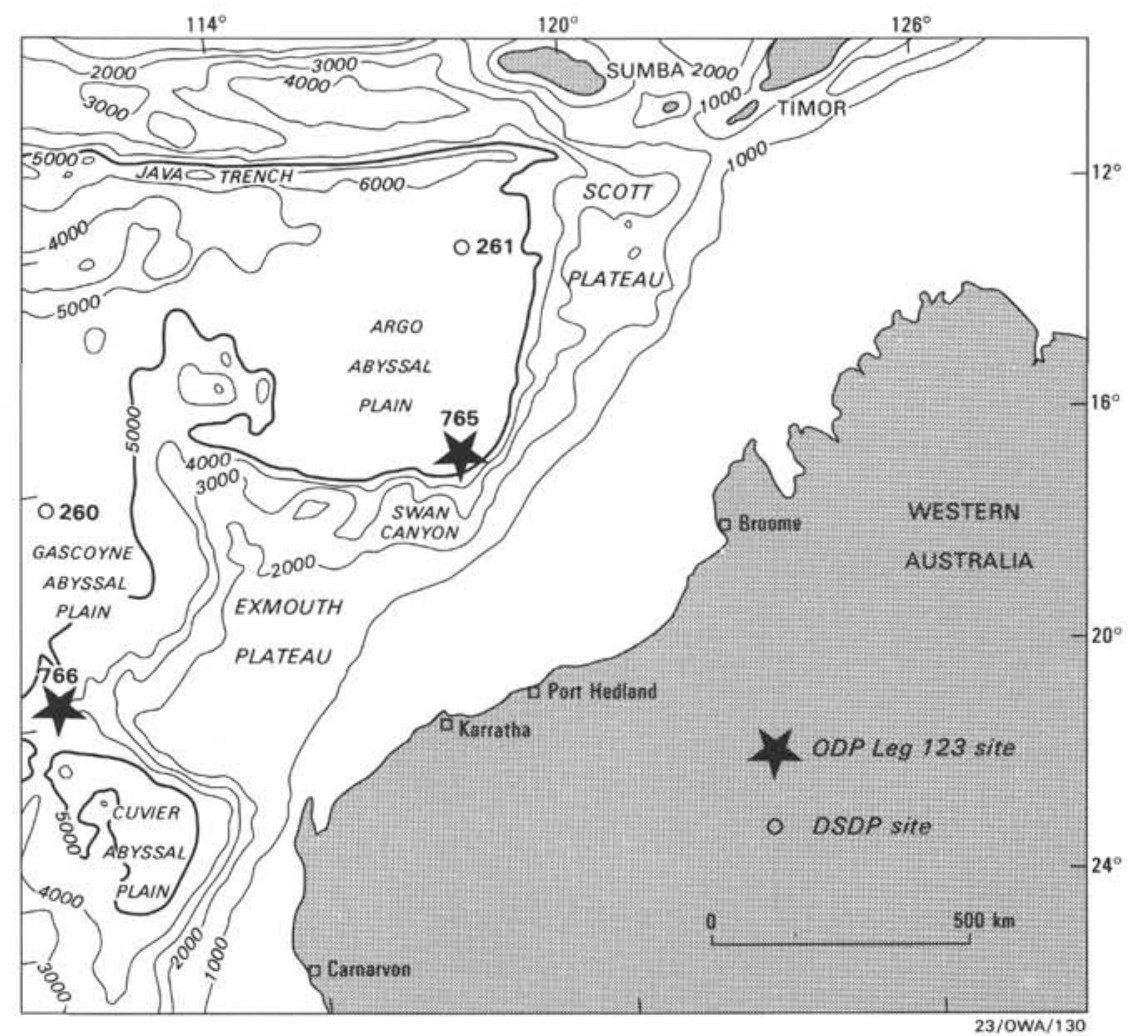

Figure 1. Map of the study area with the locations of the Argo (Site 765) and Gascoyne (Site 766) abyssal plain drill sites.

(1990). Twenty-nine samples from the Argo (Site 765) and 19 from Gascoyne (Site 766) abyssal plains were freeze dried, ground, and analyzed at the Bureau of Mineral Resources (BMR) laboratories by X-ray fluorescence (XRF) for major elements (Norrish and Hutton, 1969). The total sulfur content has been used to approximate pyrite sulfur and also to stoichiometrically calculate pyrite iron. I have assumed that organic sulfur, iron monosulfides, and oxidized sulfur species are not quantitatively important in these deep-sea sediments. These assumptions place constraints on the interpretation of the data discussed below.

Ferrous oxide was determined by a modification of the method described by Shapiro and Brannock (1961), as follows: $0.5 \mathrm{~g}$ of sediment was weighed into a $50-\mathrm{mL}$ platinum crucible and digested with $\mathrm{H}_{2} \mathrm{SO}_{4}(10 \mathrm{~mL}, 1: 3)$ and $\mathrm{HF}(5 \mathrm{~mL}, 40 \%)$ for $10 \mathrm{~min}$ on a hot plate. The digest was transferred to a $800-\mathrm{mL}$ beaker containing distilled water, boric acid, and phosphoric acid (10 $\mathrm{mL}, 1: 1)$. The solution was titrated with standard potassium dichromate solution using a Metrohm E536 potentiograph and combined platinum reference electrode. Several international standards (GSP-1, PCC-1, and BCR-1) were also analyzed, and replicate analyses of $\mathrm{BMR}$ standards indicated a precision of $1 \%-3 \%$. Although standards with sulfides were not analyzed, the method for FeO contents does not attack pyrite in the samples (G. W. Skyring, T. Donnelly, pers. comm., 1990). The mineralogy of 12 samples from the Argo Abyssal Plain was determined by X-ray diffraction (XRD).

\section{RESULTS}

\section{Calcium Carbonate and Major Elements}

The downhole calcium carbonate results are reported in Ludden, Gradstein, et al. (1990) and are shown, for both sites, in Figure 2. Erratic variations in the downhole calcium carbonate contents of the Cenozoic section from Argo Abyssal Plain (Fig.
2A) indicate turbidite-dominated sedimentation below the calcitecompensation depth (Ludden, Gradstein, et al., 1990). Calcium carbonate contents of Lower Cretaceous sediments are generally low, although some samples had calcium carbonate approaching $60 \mathrm{wt} \%$. Calcareous and siliciclastic turbidites and radiolarites are evident throughout the Cretaceous, but the Lower Cretaceous section is dominated by alternating bands of various thicknesses of red, brown, green, dark-green, dark-gray, and black claystones (von Rad et al., 1989; Ludden, Gradstein, et al., 1990).

The downhole calcium carbonate profile from Gascoyne Abyssal Plain (Fig. 2B) indicates generally low $(<15 \mathrm{wt} \%)$ calcium carbonate in the Berriasian, Valanginian, and Barremian sediments and a marked increase in calcium carbonate within the Aptian sediments. This increase in carbonate suggests a change in sedimentation near the Aptian/Barremian boundary from hemipelagic to pelagic conditions; the Lower Cretaceous sediments are combinations of dark-green calcareous claystones, sandstones, and siltstones, while the Late Cretaceous and Tertiary sediments are combinations of calcareous ooze, siliceous clays, and turbidites (von Rad et al., 1989; Ludden, Gradstein, et al., 1990).

Sample selection of the Lower Cretaceous sediments from both sites, for analyses and consideration of diagenetic products, focussed on sediments that are not significantly diluted by biogenic components so that iron limitation is not a factor influencing pyrite formation (Raiswell and Berner, 1986). The XRF major element data from Sites 765 (Argo Abyssal Plain) and 766 (Gascoyne Abyssal Plain) are summarized in Tables 1 and 2. The samples contain $<20 \mathrm{wt} \%$ calcium carbonate (estimated from $\mathrm{CaO}$ data). The XRF data show that $\mathrm{Al}_{2} \mathrm{O}_{3}$ contents vary between 4 and $16 \mathrm{wt} \%$ and $\mathrm{SiO}_{2}$ between 30 and $80 \mathrm{wt} \%$. A crossplot of these data (Fig. 3) shows decreasing $\mathrm{Al}_{2} \mathrm{O}_{3}$ with increasing $\mathrm{SiO}_{2}$, indicating some dilution of the terrigenous aluminosilicate components by another siliceous (biogenic-marine) phase, probably radiolarites. 

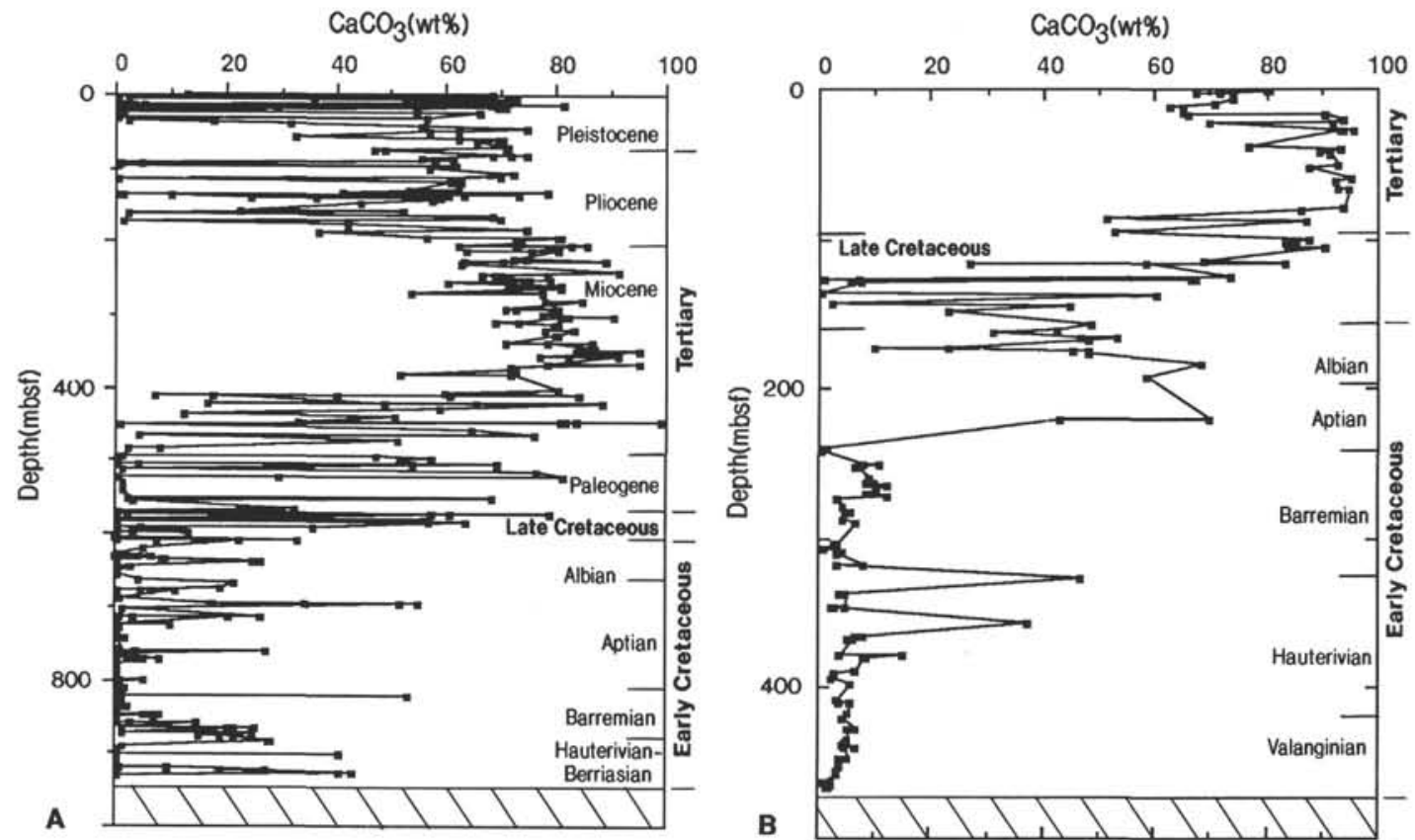

Figure 2. A. Calcium carbonate vs. depth (mbsf) for the Argo Abyssal Plain (cross-hatching = basement). B. Calcium carbonate vs. depth (mbsf) for the Gascoyne Abyssal Plain (cross-hatching = basement).

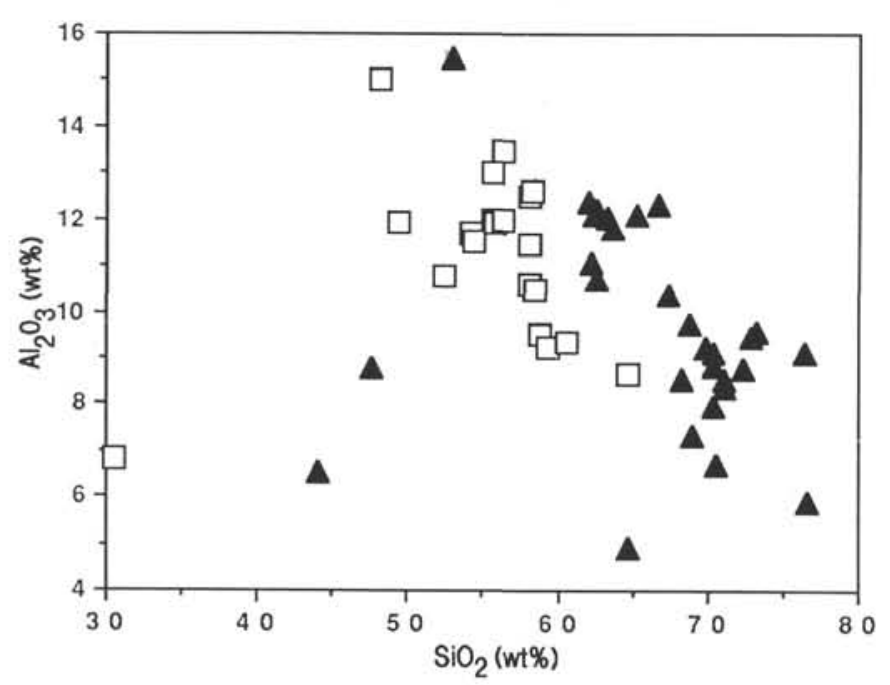

Figure 3. $\mathrm{Al}_{2} \mathrm{O}_{3}$ (wt \%) vs. $\mathrm{SiO}_{2}$ (wt \%) for Argo (closed triangles) and Gascoyne (open squares) abyssal plain sediments.

\section{Organic Matter (TOC) in Sediments}

The oxidation of organic matter within sediments results in the liberation to pore waters of oxidation/reduction metabolites, a change in the molecular composition of the organic matter, and the accumulation in the sediments of diagenetic byproducts formed by the reaction of pore-water metabolites with sedimentary components (Froelich et al., 1979; Berner, 1981).

A brief description of the sedimentary units, from which samples were collected for this work, together with TOC, TOC/total $\mathrm{S}$ (wt \%) ratios, TOC/total $\mathrm{P}($ atom) ratios, iron speciation including total iron $\mathrm{Fe}(\mathrm{t})$, ferrous iron $\mathrm{FeII}$, the ratio $\mathrm{FeII} / \mathrm{Fe}(\mathrm{t})$, pyrite iron $\mathrm{Fe}$ (py) calculated stoichiometrically from total S (Tables 1 and 2) and the ratios $\mathrm{Fe}(\mathrm{py}) / \mathrm{Fe}(\mathrm{t})$ and $[\mathrm{FeII}+\mathrm{Fe}(\mathrm{py})] / \mathrm{Fe}(\mathrm{t})$ are summarized in Tables 3 (Argo Abyssal Plain) and 4 (Gascoyne Abyssal Plain).
The TOC data for both sites are summarized in Figures 4A and 4B. Although the Lower Cretaceous sediments from both sites were visually scrutinized at sea for dark, possibly TOC-rich sediments, most samples were generally low in TOC. TOC at the Argo Abyssal Plain was highest in the Barremian-Aptian-Albian sections ( $\leq 1.1 \mathrm{wt} \%)$ near the Aptian/Albian boundary and one thin $(1 \mathrm{~cm})$ sediment layer near the Barremian/Aptian boundary had a TOC of $5.1 \mathrm{wt} \%$ (Fig. 4A). TOC in the Albian and Aptian sediments from Gascoyne Abyssal Plain (Fig. 4B) were low $(<0.1$ wt $\%)$. Relatively high TOC contents $(\leq 0.8 \mathrm{wt} \%)$ were measured in the Barremian dark-green calcareous claystones and Valanginian siltstones (1.6 wt\%) from Gascoyne Abyssal Plain (Fig. 4B). While several of the Valanginian-Hauterivian samples were $<0.5 \mathrm{wt} \%$ TOC, these were still higher than TOC in the Berriasian-Hauterivian claystones from the Argo Abyssal Plain (Fig. 4A).

The organic carbon accumulation rates in Cretaceous sediments are summarized in Table 5. The Upper Cretaceous sediments from both sites are characterized by low TOC, low sedimentation rates, and low organic-carbon accumulation rates. The Lower Cretaceous sediments, in contrast, are characterized by higher average TOC, high sedimentation rates, and higher organic-carbon accumulation rates.

\section{Ratios of Organic Carbon to Total Sulfur (C/S)}

The principal controls on pyrite formation within sediments (Berner, 1984) are (1) the amount, type, and reactivity of organic matter, (2) the amount of reactive iron minerals, and (3) the availability of dissolved sulfate. Plots of pyrite sulfur and TOC in sediments have been used to distinguish between marine and brackish-water environments and also between deposition from normal marine conditions (those underlying oxygenated bottom waters where sulfate reduction begins a few centimeters below the sediment/seawater interface) and from an anoxic water column (Berner and Raiswell, 1983; Leventhal, 1983; Berner, 1984).

Sulfate is not limiting in normal marine sediments and, where iron is not limiting, the accumulation of pyrite is controlled by the amount and reactivity of metabolizable organic carbon. In modern normal marine sediments there is a good positive correlation 
Table 1. Major element and FeO determinations, Site 765, Argo Abyssal Plain.

\begin{tabular}{|c|c|c|c|c|c|c|c|c|c|c|c|c|c|c|}
\hline $\begin{array}{l}\text { Core, section, } \\
\text { interval }(\mathrm{cm})\end{array}$ & $\begin{array}{l}\text { Depth } \\
\text { (mbsf) }\end{array}$ & $\mathrm{SiO} 2$ & $\mathrm{TiO} 2$ & $\mathrm{Al} 2 \mathrm{O} 3$ & $\mathrm{Fe} 2 \mathrm{O} 3$ & $\mathrm{FeO}$ & $\begin{array}{l}\mathrm{MnO} \\
11 \text { in wt }\end{array}$ & ${ }_{\%)}^{\mathrm{MgO}}$ & $\mathrm{CaO}$ & $\mathrm{Na} 2 \mathrm{O}$ & $\mathrm{K} 2 \mathrm{O}$ & P2O5 & $\mathrm{s}$ & LOI \\
\hline \multicolumn{15}{|l|}{$123-765 \mathrm{C}-$} \\
\hline $30 R-4,32-34$ & 630.64 & 52.94 & 0.78 & 15.48 & 7.28 & 0.21 & 0.23 & 2.51 & 2.87 & 2.22 & 3.14 & 0.11 & 0.05 & 12.19 \\
\hline $35 R-3,55-57$ & 677.65 & 70.56 & 0.35 & 6.67 & 1.84 & 0.54 & 0.02 & 1.23 & 4.02 & 1.23 & 1.00 & 0.13 & 0.30 & 12.11 \\
\hline $35 R-3,60-69$ & 677.74 & 69.02 & 0.38 & 7.32 & 2.00 & 0.66 & 0.02 & 1.34 & 5.02 & 1.26 & 1.09 & 0.14 & 0.38 & 11.36 \\
\hline $40 R-4,143-144$ & 726.83 & 64.78 & 0.85 & 4.89 & 4.89 & 0.59 & 0.05 & 2.79 & 0.63 & 1.47 & 2.06 & 0.11 & 0.04 & 9.86 \\
\hline $44 R-3,24-25$ & 761.74 & 62.60 & 0.78 & 12.22 & 6.23 & 1.03 & 0.15 & 2.40 & 0.67 & 1.43 & 2.21 & 0.10 & 0.20 & 10.00 \\
\hline $44 R-3,42-50$ & 761.95 & 63.59 & 0.76 & 11.80 & 6.10 & 1.05 & 0.04 & 2.32 & 0.60 & 1.41 & 2.16 & 0.09 & 0.25 & 9.81 \\
\hline $44 R-3,106-107$ & 762.56 & 61.94 & 0.77 & 12.40 & 5.95 & 0.90 & 0.17 & 2.29 & 0.71 & 1.41 & 2.03 & 0.12 & 0.53 & 10.79 \\
\hline $44 \mathrm{R}-3,135-136$ & 762.86 & 63.29 & 0.70 & 12.02 & 6.04 & 0.95 & 0.07 & 2.39 & 0.60 & 1.33 & 2.31 & 0.09 & 0.15 & 10.05 \\
\hline $44 R-5,2-10$ & 764.53 & 65.29 & 0.83 & 12.09 & 4.83 & 0.61 & 0.05 & 2.84 & 0.64 & 1.49 & 2.01 & 0.10 & 0.04 & 9.18 \\
\hline $45 R-3,53-55$ & 771.73 & 67.41 & 0.60 & 10.40 & 4.15 & 0.87 & 1.28 & 1.97 & 0.79 & 1.30 & 1.76 & 0.13 & 0.22 & 9.11 \\
\hline $45 \mathrm{R}-4,100-101$ & 773.70 & 43.97 & 0.55 & 6.52 & 12.55 & 1.40 & 0.80 & 1.24 & 0.57 & 0.94 & 1.08 & 0.09 & 10.16 & 20.11 \\
\hline $47 \mathrm{R}-3,55-57$ & 790.95 & 62.58 & 0.89 & 10.73 & 9.54 & 0.61 & 0.05 & 2.47 & 0.67 & 1.43 & 1.83 & 0.10 & 0.03 & 9.07 \\
\hline $48 R-4,36-37$ & 801.76 & 62.24 & 0.67 & 11.07 & 5.17 & 0.72 & 2.17 & 2.37 & 1.07 & 1.27 & 1.77 & 0.11 & 0.09 & 11.29 \\
\hline $49 \mathrm{R}-1,94-95$ & 807.24 & 72.89 & 0.47 & 9.47 & 2.76 & 0.56 & 0.03 & 1.82 & 0.53 & 1.06 & 1.50 & 0.09 & 0.24 & 8.57 \\
\hline $49 \mathrm{R}-1,96-104$ & 807.28 & 73.32 & 0.48 & 9.57 & 2.79 & 0.58 & 0.17 & 1.86 & 0.58 & 1.10 & 1.55 & 0.10 & 0.25 & 7.65 \\
\hline $50 \mathrm{R}-2,80-82$ & 817.90 & 69.88 & 0.84 & 9.21 & 4.89 & 0.69 & 0.14 & 2.33 & 0.79 & 1.20 & 1.75 & 0.23 & 0.09 & 7.95 \\
\hline $50 \mathrm{R}-6,51-52$ & 823.61 & 70.38 & 0.79 & 8.80 & 4.71 & 0.61 & 0.04 & 2.12 & 0.55 & 1.18 & 1.64 & 0.08 & 0.06 & 9.05 \\
\hline 51R-CC. $2-5$ & 832.52 & 70.34 & 0.68 & 9.13 & 4.29 & 0.77 & 0.04 & 2.29 & 0.54 & 1.25 & 1.78 & 0.09 & 0.18 & 8.62 \\
\hline $52 \mathrm{R}-1,132-133$ & 835.82 & 71.04 & 0.63 & 8.53 & 4.37 & 0.80 & 0.04 & 2.26 & 0.50 & 1.13 & 1.83 & 0.07 & 0.16 & 8.62 \\
\hline $52 \mathrm{R}-2,8-10$ & 836.08 & 76.51 & 0.69 & 9.13 & 4.47 & 0.69 & 0.07 & 2.35 & 0.54 & 1.11 & 1.90 & 0.08 & 0.16 & 2.30 \\
\hline $52 \mathrm{R}-2,10-11$ & 836.10 & 71.11 & 0.63 & 8.37 & 4.19 & 0.82 & 0.14 & 2.21 & 0.53 & 1.12 & 1.63 & 0.07 & 0.08 & 9.09 \\
\hline $53 \mathrm{R}-3,72-74$ & 847.82 & 70.42 & 0.50 & 7.96 & 5.85 & 0.27 & 0.13 & 2.16 & 0.56 & 1.07 & 1.40 & 0.10 & 0.09 & 9.47 \\
\hline $53 \mathrm{R}-6,123-125$ & 852.83 & 76.68 & 0.36 & 5.90 & 4.61 & 0.18 & 0.09 & 1.58 & 0.46 & 0.89 & 1.20 & 0.12 & 0.10 & 7.84 \\
\hline $55 \mathrm{R}-2,82-84$ & 865.32 & 68.30 & 0.55 & 8.51 & 5.87 & 0.29 & 0.56 & 2.37 & 0.97 & 1.09 & 1.56 & 0.26 & 0.16 & 9.52 \\
\hline $57 \mathrm{R}-7,15-17$ & 890.55 & 72.35 & 0.43 & 8.78 & 5.93 & 0.24 & 0.55 & 2.17 & 0.76 & 1.14 & 1.49 & 0.13 & 0.08 & 5.94 \\
\hline $59 \mathrm{R}-3,123-125$ & 902.13 & 68.80 & 0.42 & 9.77 & 5.62 & 0.28 & 0.04 & 2.38 & 0.74 & 1.13 & 1.32 & 0.13 & 0.07 & 9.31 \\
\hline $59 \mathrm{R}-4,111-112$ & 903.51 & 66.67 & 0.66 & 12.30 & 2.16 & 0.40 & 0.03 & 2.58 & 0.74 & 1.22 & 1.89 & 0.11 & 0.13 & 11.12 \\
\hline $60 R-2,61-63$ & 909.61 & 62.41 & 0.55 & 12.08 & 8.10 & 0.25 & 0.13 & 2.43 & 0.77 & 1.10 & 2.88 & 0.19 & 0.04 & 9.08 \\
\hline $61 R-5,105-106$ & 923.95 & 47.61 & 0.67 & 8.76 & 7.86 & 0.00 & 1.46 & 1.96 & 10.77 & 0.94 & 2.57 & 0.14 & 0.03 & 17.25 \\
\hline
\end{tabular}

Table 2. Major element and FeO determinations, Site 766, Gascoyne Abyssal Plain.

\begin{tabular}{|c|c|c|c|c|c|c|c|c|c|c|c|c|c|c|}
\hline $\begin{array}{l}\text { Core, section, } \\
\text { interval }(\mathrm{cm})\end{array}$ & $\begin{array}{l}\text { Depth } \\
\text { (mbsf) }\end{array}$ & $\mathrm{SiO} 2$ & $\mathrm{TiO} 2$ & $\mathrm{Al} 2 \mathrm{O} 3$ & $\mathrm{Fe} 2 \mathrm{O} 3$ & $\mathrm{FeO}_{\text {(a }}$ & $\begin{array}{l}\mathrm{MnO} \\
11 \text { in wt? }\end{array}$ & $\mathrm{MgO}$ & $\mathrm{CaO}$ & $\mathrm{Na} 2 \mathrm{O}$ & $\mathrm{K} 2 \mathrm{O}$ & $\mathrm{P} 2 \mathrm{O} 5$ & $\mathrm{~S}$ & LOI \\
\hline \multicolumn{15}{|l|}{$123-766 \mathrm{~A}-$} \\
\hline $14 R-4,7-8$ & 128.17 & 49.42 & 0.86 & 11.94 & 6.93 & 0.00 & 0.47 & 4.14 & 3.73 & 1.91 & 2.43 & 0.34 & 0.24 & 17.59 \\
\hline $17 \mathrm{R}-6,105-106$ & 161.05 & 30.61 & 0.64 & 6.81 & 4.73 & 0.00 & 0.83 & 1.66 & 24.46 & 1.65 & 1.40 & 0.17 & 0.07 & 26.97 \\
\hline $28 \mathrm{R}-2,25-28$ & 260.35 & 58.74 & 0.45 & 9.49 & 3.68 & 0.58 & 0.02 & 2.30 & 5.28 & 1.74 & 1.47 & 0.09 & 0.41 & 15.75 \\
\hline $29 \mathrm{R}-2,44-46$ & 270.24 & 60.62 & 0.39 & 9.36 & 3.68 & 0.70 & 0.02 & 2.24 & 4.92 & 1.51 & 1.45 & 0.12 & 0.62 & 14.36 \\
\hline $30 \mathrm{R}-4,41-44$ & 282.81 & 58.10 & 0.63 & 11.44 & 4.57 & 0.93 & 0.02 & 2.69 & 3.41 & 1.75 & 2.02 & 0.16 & 0.85 & 13.44 \\
\hline $32 \mathrm{R}-4,138-141$ & 303.08 & 64.74 & 0.66 & 8.63 & 4.82 & 1.01 & 0.01 & 1.94 & 2.11 & 1.48 & 2.28 & 0.11 & 1.16 & 11.04 \\
\hline $34 \mathrm{R}-1,75-76$ & 317.25 & 59.40 & 0.77 & 9.23 & 5.80 & 1.31 & 0.02 & 2.07 & 4.64 & 1.42 & 3.03 & 0.16 & 0.23 & 11.92 \\
\hline $36 \mathrm{R}-1,138-139$ & 337.18 & 58.07 & 0.97 & 10.62 & 6.03 & 1.54 & 0.02 & 2.63 & 2.75 & 1.60 & 3.86 & 0.14 & 0.31 & 11.47 \\
\hline $37 \mathrm{R}-1,69-70$ & 346.19 & 58.39 & 0.95 & 10.46 & 5.78 & 1.52 & 0.02 & 2.52 & 2.84 & 1.41 & 3.36 & 0.14 & 0.73 & 11.89 \\
\hline $39 \mathrm{R}-2,122-124$ & 367.52 & 55.57 & 1.06 & 11.98 & 5.18 & 1.70 & 0.02 & 2.88 & 3.26 & 1.45 & 3.48 & 0.14 & 0.45 & 12.83 \\
\hline $39 \mathrm{R}-3,32-34$ & 368.12 & 54.33 & 1.04 & 11.51 & 6.00 & 2.13 & 0.02 & 3.19 & 4.47 & 1.88 & 3.25 & 0.14 & 0.27 & 11.77 \\
\hline $39 \mathrm{R}-3,32-34$ & 368.12 & 55.78 & 1.05 & 11.90 & 6.16 & 2.14 & 0.02 & 3.26 & 4.12 & 1.61 & 3.37 & 0.14 & 0.27 & 10.17 \\
\hline $42 \mathrm{R}-1,94-97$ & 394.74 & 48.11 & 1.14 & 15.01 & 9.57 & 5.38 & 0.03 & 3.55 & 1.81 & 1.64 & 2.29 & 0.09 & 0.16 & 11.24 \\
\hline $45 R-4,106-108$ & 428.36 & 56.30 & 1.00 & 11.98 & 4.60 & 2.53 & 0.02 & 2.32 & 3.15 & 1.39 & 2.33 & 0.16 & 1.97 & 12.25 \\
\hline $46 \mathrm{R}-3,32-34$ & 435.86 & 58.09 & 0.90 & 12.50 & 4.79 & 1.53 & 0.01 & 2.31 & 2.89 & 1.49 & 2.52 & 0.15 & 1.10 & 11.71 \\
\hline $46 R-5,141-143$ & 439.95 & 54.14 & 0.94 & 11.71 & 5.57 & 1.89 & 0.03 & 2.21 & 4.22 & 1.47 & 2.28 & 0.17 & 2.17 & 13.19 \\
\hline $46 \mathrm{R}-6,58-59$ & 440.62 & 56.38 & 1.07 & 13.50 & 4.88 & 1.78 & 0.02 & 2.51 & 3.05 & 1.55 & 2.61 & 0.19 & 0.96 & 11.50 \\
\hline $47 \mathrm{R}-3,103-105$ & 446.23 & 55.70 & 0.95 & 13.01 & 4.56 & 2.25 & 0.02 & 2.47 & 3.54 & 1.45 & 2.59 & 0.27 & 0.87 & 12.32 \\
\hline $49 \mathrm{R}-1,97-98$ & 462.47 & 52.46 & 0.80 & 10.80 & 6.99 & 6.11 & 0.03 & 7.80 & 0.89 & 1.54 & 1.92 & 0.12 & 0.48 & 10.05 \\
\hline $49 \mathrm{R}-2,142-143$ & 464.42 & 58.37 & 0.87 & 12.60 & 5.64 & 1.87 & 0.02 & 3.23 & 1.74 & 1.28 & 4.11 & 0.22 & 1.04 & 9.05 \\
\hline
\end{tabular}

between organic carbon and pyrite sulfur with a line of best-fit extending through the origin with a mean $\mathrm{C} / \mathrm{S}$ ratio of $\sim 2.8$ (Leventhal, 1983; Berner and Raiswell, 1983; Berner, 1984; Raiswell and Berner, 1986). However, in fresh or brackish waters, where sulfate may be limiting or organic matter that is unreactive to the sulfate-reducing bacteria is present, the amount of pyrite formed is less than that formed in marine sediments for comparable quantities of organic carbon buried. Hence, C/S ratios are higher than 2.8 , depending upon the salinity and sulfate content of the overlying water as well as the type and reactivity of the organic matter buried in the sediments (Berner and Raiswell, 1984).

$\mathrm{C} / \mathrm{S}$ ratios have been used to comment upon depositional environments from other Australian sediments, including the Cretaceous Toolebuc Formation (Boreham and Powell, 1987), the middle Cambrian sediments of the Georgina Basin (Donnelly et al., 1988) and the middle Proterozoic Velkerri Formation in the McArthur Basin (Donnelly and Crick, 1988). 
Table 3. TOC and diagenetic products, Site 765, Argo Abyssal Plain.

\begin{tabular}{|c|c|c|c|c|c|c|c|c|c|c|c|c|c|}
\hline $\begin{array}{l}\text { Core, section, } \\
\text { interval }(\mathrm{cm})\end{array}$ & $\begin{array}{l}\text { Depth } \\
\text { (mbsf) }\end{array}$ & Age & Color & Unit & $\begin{array}{l}\mathrm{Fe}(\mathrm{t}) \\
(\mathrm{wt} \%)\end{array}$ & $\begin{array}{c}\text { Fell } \\
(w t \%)\end{array}$ & $\mathrm{FeII} / \mathrm{Fe}(\mathrm{t})$ & $\begin{array}{l}\mathrm{Fe} \text { (py) } \\
\text { (wt\%) }\end{array}$ & $\begin{array}{c}\mathrm{Fe}(\mathrm{py}) / \mathrm{Fe}(\mathrm{t}) \\
\text { (DOP) }\end{array}$ & $\Sigma \mathrm{Fe} / \mathrm{Fe}(\mathrm{t})$ & $\begin{array}{c}\text { TOC } \\
\text { (wt\%) }\end{array}$ & $\begin{array}{l}\mathrm{C} / \mathrm{S} \\
\text { (wt) }\end{array}$ & $\underset{\text { (atom) }}{\mathrm{C} / \mathrm{P}}$ \\
\hline \multicolumn{14}{|l|}{$123-765 \mathrm{C}-$} \\
\hline $30 \mathrm{R}-4,32-34$ & 630.64 & $\mathrm{Al}$ & orange & IVB & 5.26 & 0.16 & 0.03 & 0.04 & 0.01 & 0.04 & 0.00 & & \\
\hline $35 \mathrm{R}-3,55-57$ & 677.65 & $\mathrm{~A}$ & green & IVC & 1.71 & 0.42 & 0.25 & 0.26 & 0.15 & 0.40 & 1.08 & 3.60 & 4.91 \\
\hline $35 R-3,60-69$ & 677.74 & A & green & IVC & 1.91 & 0.51 & 0.27 & 0.33 & 0.17 & 0.44 & 0.35 & 0.92 & 1.48 \\
\hline $40 \mathrm{R}-4,143-144$ & 726.83 & $\mathrm{eA}$ & green & VA & 3.88 & 0.46 & 0.12 & 0.04 & 0.01 & 0.13 & 0.44 & 11.00 & 2.36 \\
\hline $44 \mathrm{R}-3,24-25$ & 761.74 & $\mathrm{eA}$ & gray/green & VB & 5.16 & 0.80 & 0.16 & 0.18 & 0.03 & 0.19 & 0.36 & 1.80 & 2.13 \\
\hline $44 \mathrm{R}-3,42-50$ & 761.95 & $\mathrm{eA}$ & gray/green & VB & 5.09 & 0.82 & 0.16 & 0.22 & 0.04 & 0.20 & 1.23 & 4.92 & 8.08 \\
\hline $44 \mathrm{R}-3,106-107$ & 762.56 & $\mathrm{eA}$ & gray/green & VB & 4.86 & 0.70 & 0.14 & 0.46 & 0.10 & 0.24 & 0.46 & 0.87 & 2.27 \\
\hline $44 \mathrm{R}-3,135-136$ & 762.86 & $\mathrm{eA}$ & gray/green & VB & 4.97 & 0.74 & 0.15 & 0.13 & 0.03 & 0.18 & 0.43 & 2.87 & 2.82 \\
\hline $44 R-5,2-10$ & 764.53 & $\mathrm{eA}$ & green & VB & 3.85 & 0.47 & 0.12 & 0.04 & 0.01 & 0.13 & 0.40 & 10.00 & 2.36 \\
\hline $45 R-3,53-55$ & 771.73 & $\mathrm{eA}$ & gray/green & VB & 3.58 & 0.68 & 0.19 & 0.19 & 0.05 & 0.24 & 0.24 & 1.09 & 1.09 \\
\hline $45 R-4,100-101$ & 773.70 & $\mathrm{eA}$ & black & VB & 9.87 & 1.09 & 0.11 & 8.89 & 0.90 & 1.01 & 5.12 & 0.50 & 33.62 \\
\hline $47 R-3,55-57$ & 790.95 & $\mathrm{eA}$ & red & VB & 7.15 & 0.47 & 0.07 & 0.03 & 0.00 & 0.07 & 0.04 & 1.33 & 0.24 \\
\hline $48 R-4,36-37$ & 801.76 & $\mathrm{eA}$ & green & VB & 4.18 & 0.56 & 0.13 & 0.08 & 0.02 & 0.15 & 0.05 & 0.56 & 0.27 \\
\hline $49 \mathrm{R}-1,94-95$ & 807.24 & B & green & VC & 2.37 & 0.44 & 0.19 & 0.21 & 0.09 & 0.27 & 0.57 & 2.38 & 3.74 \\
\hline $50 \mathrm{R}-6,51-52$ & 823.61 & $\mathrm{~B}$ & gray/green & VC & 3.77 & 0.47 & 0.12 & 0.05 & 0.01 & 0.14 & 0.31 & 5.17 & 2.29 \\
\hline $51 \mathrm{R}-\mathrm{CC}, 2-5$ & 832.52 & B & green & VC & 3.60 & 0.60 & 0.17 & 0.16 & 0.04 & 0.21 & 0.28 & 1.56 & 1.84 \\
\hline $52 \mathrm{R}-1,132-133$ & 835.82 & B & green & VC & 3.68 & 0.62 & 0.17 & 0.14 & 0.04 & 0.21 & 0.33 & 2.06 & 2.79 \\
\hline $52 \mathrm{R}-2,8-10$ & 836.08 & B & green & VC & 3.67 & 0.54 & 0.15 & 0.14 & 0.04 & 0.19 & 0.23 & 1.44 & 1.70 \\
\hline $52 \mathrm{R}-2,10-11$ & 836.10 & B & gray/green & VC & 3.57 & 0.64 & 0.18 & 0.07 & 0.02 & 0.20 & 0.28 & 3.50 & 2.36 \\
\hline $53 R-3,72-74$ & 847.82 & B & red & VC & 4.30 & 0.21 & 0.05 & 0.08 & 0.02 & 0.07 & 0.02 & 0.22 & 0.12 \\
\hline $53 \mathrm{R}-6,123-125$ & 852.83 & B & red & VC & 3.37 & 0.14 & 0.04 & 0.09 & 0.03 & 0.07 & 0.00 & & \\
\hline $55 R-2,82-84$ & 865.32 & $\mathrm{H}$ & red & VI & 4.33 & 0.23 & 0.05 & 0.14 & 0.03 & 0.09 & 0.22 & 1.38 & 0.50 \\
\hline $57 \mathrm{R}-7,15-17$ & 890.55 & $\mathrm{~V} / \mathrm{H}$ & red & VI & 4.34 & 0.19 & 0.04 & 0.07 & 0.02 & 0.06 & 0.01 & 0.13 & 0.05 \\
\hline $59 \mathrm{R}-3,123-125$ & 902.13 & $\mathrm{~B} / \mathrm{V}$ & red & VII & 4.15 & 0.22 & 0.05 & 0.06 & 0.02 & 0.07 & 0.03 & 0.43 & 0.14 \\
\hline $59 \mathrm{R}-4,111-112$ & 903.51 & $\mathrm{~B} / \mathrm{V}$ & green & VII & 1.82 & 0.31 & 0.17 & 0.11 & 0.06 & 0.23 & 0.50 & 3.85 & 2.69 \\
\hline $60 \mathrm{R}-2,61-63$ & 909.61 & $\mathrm{~B} / \mathrm{V}$ & red & VII & 5.86 & 0.19 & 0.03 & 0.04 & 0.01 & 0.04 & 0.02 & 0.50 & 0.06 \\
\hline $61 \mathrm{R}-5,105-106$ & 923.95 & B & red & VII & 5.50 & 0.00 & 0.00 & 0.03 & 0.01 & 0.00 & 0.00 & & \\
\hline
\end{tabular}

Age; $\mathrm{Al}=$ Albian, $\mathrm{A}=$ Aptian, $\mathrm{eA}=$ early Aptian, $\mathrm{B}=$ Barremian, $\mathrm{V}=$ Valanginian, $\mathrm{H}=$ Hauterivian

$\mathrm{Fe}(\mathrm{t})=$ total iron, $\mathrm{Fell}=$ ferrous iron, $\mathrm{Fe}(\mathrm{py})=$ pyrite iron, $\Sigma \mathrm{Fe}=$ ferrous + pyrite iron, TOC all in wt\%. $\mathrm{Fe}(\mathrm{py}) / \mathrm{Fe}(\mathrm{t})=$ degree of pyritization $(\mathrm{DOP})$.

Brief descriptions of the subunits from which samples were collected are included below. A complete description can be found in Ludden, Gradstein, et al. (1990). Subunit IVB: late Albian to late Aptian light colored clays and minor calcareous turbidites. Subunit IVC: late Aptian light to minor dark claystones, lesser calcareous turbidites, and minor radiolarites. Subunit VA: early Aptian dark claystones with minor laminated silty layers. Subunit VB: Barremian to early Aptian dark claystones with laminated silty layers; color extremely variable but predominantly gray to very dark gray, dark reddish-brown, dark reddish-gray, dusky green or grayish green. Color banding occurs on a cm-dm scale, elongated and flattened mottles indicate diagenetic processes and or bioturbation, scattered occurrences of pyrite, zeolites, and organic debris observed in smear slides. Rhodochrosite sediment and /or microconcretions occur throughout the subunit. Subunit VC: Barremian reddish-brown and green claystones, lesser rhodochrosite and radiolarites, similar color banding as for Subunit VB, closely spaced bands of alternating colors are common, probably flattened burrows. Rhodochrosite micronodules and pyrite are sparse. Unit VI: Valanginian to Hauterivian reddish-brown chalks (turbidites), lesser claystones and minor radiolarites, calcareous claystones constitute about two-thirds of Unit VI and are typically reddish-brown and less commonly greenish-gray, locally abundant bioturbation, some rhodochrosite micronodules, and minor amounts of organic matter and pyrite, glauconite and phosphatic debris evident. Unit VII: Berriasian to Valanginian reddish-brown and green claystone, minor radiolarites and bentonites and brown silty claystones, claystones contain minor silt or sand, rhodochrosite micronodules, radiolarians, organic debris, pyrite, apatite, and feldspar.

\section{Total Organic Carbon (TOC) and Sulphur (S)}

$\mathrm{C} / \mathrm{S}$ ratios determined on individual samples from both sites are summarized in Tables 3 and 4 and these data plotted in Figure 5. Total sulfur has been used to approximate pyrite sulfur and individual pyrite sulfur values have not been determined. Some sulfur could be present as organic sulfur, although in these low TOC sediments organic sulfur would be expected to be small. Saxby (1986), for example, examined sulfur speciation in cores from the Eromanga Basin of eastern Australia and his data indicate that at $<2 \mathrm{wt} \%$ TOC, $80 \%-100 \%$ of total sulfur consistently occurred as pyrite. Over a wide range of organic carbon contents, $\leq \sim 10 \mathrm{wt} \%$ pyrite sulfur still represented $60 \%-85 \%$ of total sulfur, and organic sulfur contents showed no clear relationship with organic carbon concentrations. Furthermore, there is no evidence of sulfate minerals in these deep-sea sediments and residual pore water sulfate in the samples was estimated to be $<0.2 \mathrm{wt} \%$ total sulfur. Therefore, total sulfur has been used to approximate pyrite sulfur, recognizing that actual pyrite sulfur must be less than that reported from total $\mathrm{S}$ contents.
Undoubtedly, a large error is associated with $\mathrm{C} / \mathrm{S}$ ratios at low TOC contents (Raiswell and Berner, 1986). Low TOC $(<0.2 \mathrm{wt} \%)$ samples from the Argo Abyssal Plain have low total S ( $<0.1 \mathrm{wt} \%)$, $\mathrm{C} / \mathrm{S}$ ratios between $<0.3$ and 1.4 , and occur as red to red/brown sediments (Table 3 ). Excluding those low TOC samples, there remain large variations in the $\mathrm{C} / \mathrm{S}$ ratios of individual samples from all sedimentary units of Site 765 . C/S ratios vary between 0.5 for the highest TOC sample ( $5.1 \mathrm{wt} \% ; 773.7 \mathrm{mbsf})$ of early Aptian age and 11.0 , for another sample (TOC $=0.44 \mathrm{wt} \% ; 726.83$ mbsf) also of early Aptian age. For those samples with TOC $>0.2$ $\mathrm{wt} \%$, the average of individual $\mathrm{C} / \mathrm{S}$ ratios for the Aptian (sedimentary Units IVC, VA, VB) is $3.3 \pm 3.6$ (12 samples) and for Barremian samples (Subunit VC), the average of individual $\mathrm{C} / \mathrm{S}$ ratios is $2.2 \pm 1.5$ ( 9 samples). TOC and S are poorly correlated in the Lower Cretaceous sediments of Site 765; a linear regression of all that data with $\mathrm{TOC}<2 \mathrm{wt} \%$ (Fig. 5) indicates a mean $\mathrm{C} / \mathrm{S}$ ratio of $5.4(\mathrm{R}=0.48)$.

TOC data and $\mathrm{C} / \mathrm{S}$ ratios from Gascoyne Abyssal Plain are summarized in Table 4. TOC and total S are plotted in Figure 5. For those samples with TOC $>0.2 \mathrm{wt} \%$, the average of individual 
Table 4. TOC and diagenetic products, Site 766, Gascoyne Abyssal Plain.

\begin{tabular}{|c|c|c|c|c|c|c|c|c|c|c|c|c|c|}
\hline $\begin{array}{l}\text { Core, section, } \\
\text { interval }(\mathrm{cm})\end{array}$ & $\begin{array}{l}\text { Depth } \\
\text { (mbsf) }\end{array}$ & Age & Color & Unit & $\begin{array}{l}\mathrm{Fe}(\mathrm{t}) \\
(\mathrm{wt} \%)\end{array}$ & $\begin{array}{c}\text { Fell } \\
(w t \%)\end{array}$ & $\mathrm{FeII} / \mathrm{Fe}(\mathrm{t})$ & $\begin{array}{l}\mathrm{Fe}(\mathrm{py}) \\
(w \mathrm{t} \%)\end{array}$ & $\begin{array}{c}\mathrm{Fe}(\mathrm{py}) / \mathrm{Fe}(\mathrm{t}) \\
(\mathrm{DOP})\end{array}$ & $\Sigma \mathrm{Fe} / \mathrm{Fe}(\mathrm{t})$ & $\begin{array}{c}\text { TOC } \\
(w t \%)\end{array}$ & $\begin{array}{l}\mathrm{C} / \mathrm{S} \\
(\mathrm{wt})\end{array}$ & $\begin{array}{c}\mathrm{C} / \mathrm{P} \\
\text { (atom) }\end{array}$ \\
\hline \multicolumn{14}{|l|}{$123-766 \mathrm{~A}-$} \\
\hline $14 \mathrm{R}-4,7-8$ & 128.17 & $\mathrm{~T}$ & red/brown & IIB & 4.85 & 0.00 & 0.00 & 0.21 & 0.04 & 0.04 & 0.01 & 0.04 & 0.02 \\
\hline $17 R-6,105-106$ & 161.05 & $\mathrm{Al}$ & cream & IIC & 3.31 & 0.00 & 0.00 & 0.06 & 0.02 & 0.02 & 0.00 & 0.00 & \\
\hline $28 \mathrm{R}-2,25-28$ & 260.35 & B & It green & IIIA & 3.03 & 0.45 & 0.15 & 0.36 & 0.12 & 0.27 & 0.14 & 0.34 & 9.17 \\
\hline $29 \mathrm{R}-2,44-46$ & 270.24 & B & It green & IIIA & 3.12 & 0.54 & 0.17 & 0.54 & 0.17 & 0.35 & 0.39 & 0.63 & 19.23 \\
\hline $30 \mathrm{R}-4,41-44$ & 282.81 & B & It green & IIIA & 3.92 & 0.72 & 0.18 & 0.74 & 0.19 & 0.37 & 0.58 & 0.68 & 21.28 \\
\hline $32 \mathrm{R}-4,138-141$ & 303.08 & B & dark green & IIIA & 4.16 & 0.78 & 0.19 & 1.02 & 0.25 & 0.43 & 0.71 & 0.61 & 38.46 \\
\hline $34 \mathrm{R}-1,75-76$ & 317.25 & $\mathrm{H}$ & It green & IIIB & 5.08 & 1.02 & 0.20 & 0.20 & 0.04 & 0.24 & 0.13 & 0.57 & 4.81 \\
\hline $36 \mathrm{R}-1,138-139$ & 337.18 & $\mathrm{H}$ & dark green & IIIB & 5.42 & 1.20 & 0.22 & 0.27 & 0.05 & 0.27 & 0.23 & 0.74 & 9.71 \\
\hline $37 R-1,69-70$ & 346.19 & $\mathrm{H}$ & dark green & IIIB & 5.23 & 1.18 & 0.23 & 0.64 & 0.12 & 0.35 & 0.57 & 0.78 & 23.81 \\
\hline $39 \mathrm{R}-2,122-124$ & 367.52 & $\mathrm{H}$ & dark green & IIIB & 4.95 & 1.32 & 0.27 & 0.39 & 0.08 & 0.35 & 0.46 & 1.02 & 19.61 \\
\hline $39 \mathrm{R}-3,32-34$ & 368.12 & H & dark green & IIIB & 5.86 & 1.66 & 0.28 & 0.24 & 0.04 & 0.32 & 0.07 & 0.26 & 2.96 \\
\hline $42 \mathrm{R}-1,94-97$ & 394.74 & $\mathrm{H}$ & dark green & IIIB & 10.88 & 4.18 & 0.38 & 0.14 & 0.01 & 0.40 & 0.03 & 0.19 & 1.97 \\
\hline $45 R-4,106-108$ & 428.36 & $\mathrm{H}$ & dark green & IIIB & 5.19 & 1.97 & 0.38 & 1.72 & 0.33 & 0.71 & 1.60 & 0.81 & 58.82 \\
\hline $46 \mathrm{R}-3,32-34$ & 435.86 & V & green/gray & IIIB & 4.54 & 1.19 & 0.26 & 0.96 & 0.21 & 0.47 & 0.79 & 0.72 & 31.25 \\
\hline $46 \mathrm{R}-5,141-143$ & 439.95 & V & green/gray & IIIB & 5.37 & 1.47 & 0.27 & 1.90 & 0.35 & 0.63 & 1.53 & 0.71 & 52.63 \\
\hline $46 \mathrm{R}-6,58-59$ & 440.62 & V & green/gray & IIIB & 4.80 & 1.38 & 0.29 & 0.84 & 0.18 & 0.46 & 0.69 & 0.72 & 21.28 \\
\hline $47 R-3,103-105$ & 446.23 & V & dark green & IIIB & 4.94 & 1.75 & 0.35 & 0.76 & 0.15 & 0.51 & 0.66 & 0.76 & 14.49 \\
\hline $49 R-1,97-98$ & 462.47 & V & dark green & IIIB & 9.64 & 4.75 & 0.49 & 0.42 & 0.04 & 0.54 & 0.67 & 1.40 & 33.33 \\
\hline $49 \mathrm{R}-2,142-143$ & 464.42 & V & dark green & IIIB & 5.40 & 1.45 & 0.27 & 0.91 & 0.17 & 0.44 & 0.82 & 0.79 & 22.22 \\
\hline
\end{tabular}

$\mathrm{Fe}(\mathrm{t})=$ total iron, $\mathrm{FeII}=$ ferrous iron, $\mathrm{Fe}(\mathrm{py})=$ pyrite iron, $\Sigma \mathrm{Fe}=$ ferrous + pyrite iron, $\mathrm{TOC}$ all in $w \mathrm{t} \% . \mathrm{Fe}(\mathrm{py}) / \mathrm{Fe}(\mathrm{t})=$ degree of pyritization $(\mathrm{DOP})$.

Age: $\mathrm{T}=$ Turonian, $\mathrm{Al}=$ Albian, $\mathrm{B}=$ Barremian, $\mathrm{H}=$ Hauterivian, $\mathrm{V}=$ Valanginian

Brief descriptions of the subunits from which samples were collected; a complete description can be found in Ludden, Gradstein, et al. (1990). Unit IIB: calcareous ooze and clays are interlaminated, a distinctively color-banded interval. Unit IIC: dominated by bioturbated zeolitic, clayey nannofossil ooze intercalated with lighter brown nannofossil ooze and chalk. Unit IIIA: claystones with disseminated glauconite, quartz and radiolarians; bioturbation is pervasive, pyrite nodules occur sporadically. A large pyrite nodule coated with cubes and pyritohedrons of pyrite was found in this subunit, Section $123-766 \mathrm{~A}-31 \mathrm{R}-1$ at $15-21 \mathrm{~cm}$. Unit IIIB: sandstones and siltstones containing various proportions of altered volcanic lithic grains, glauconite and neritic carbonate bioclasts, dark to very dark greenish-gray sandstones constitute the upper part of Subunit IIB, sandy to clayey siltstones predominate in the lower part. Bioturbation is pervasive, pyrite concretions occur throughout, pyrite is particularly abundant in the lower part of the Subunit in the sandy to clayey siltstones, other components noted are coal fragments, radiolarians, and feldspar.
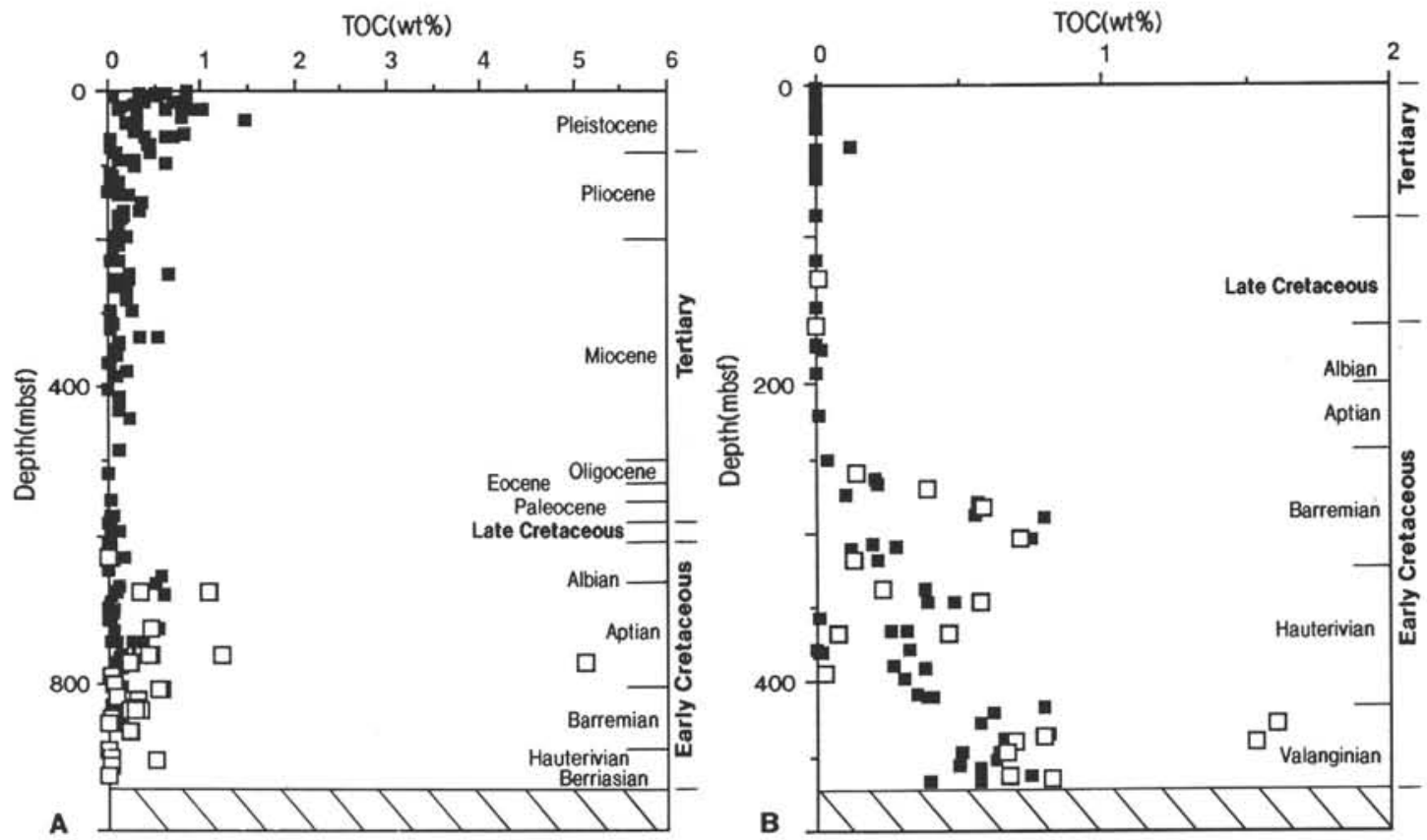

Figure 4. A. TOC vs. depth (mbsf) Argo Abyssal Plain (Site 765). B. TOC vs. depth (mbsf) Gascoyne Abyssal Plain (Site 766).

Open squares $=$ samples analyzed for this study.

$\mathrm{C} / \mathrm{S}$ ratios for Hauterivian/Valanginian sediments (Unit IIIB) is $0.7 \pm 0.3$ (13 samples) and for Barremian/lower Aptian sediments (Units IIC, IIIA) $0.6 \pm 0.2$ ( 5 samples), while a linear regression to all the Site 766 data in Figure 5 indicates a mean $\mathrm{C} / \mathrm{S}$ of $0.8(\mathrm{R}=$ $0.97)$.
A comparison of the data from the two sites shows consistently higher quantities of pyrite in Site 766 sediments than in those at Site 765 , for comparable quantities of TOC buried. The result suggests that a more metabolizable organic matter, reactive to the sulfate-reducing bacteria, is buried in the Gascoyne Abyssal Plain 
Table 5. Average organic carbon accumulation rates, Argo and Gascoyne abyssal plains.

\begin{tabular}{ccccc}
\hline Core & Age & $\begin{array}{c}\text { TOC } \\
(\mathrm{wt} \%)\end{array}$ & $\begin{array}{c}\text { Sed. rate } \\
(\mathrm{m} / \mathrm{m} . \mathrm{y} .)\end{array}$ & $\begin{array}{c}\text { TOC accumulation } \\
\left(\mathrm{g} / \mathrm{cm}^{2} / \mathrm{m} . \mathrm{y} .\right)\end{array}$ \\
\hline
\end{tabular}

Argo Abyssal Plain

$123-765 \mathrm{C}-$

$\begin{array}{lllcr}48 \mathrm{R}-62 \mathrm{R} & \mathrm{Be}-\mathrm{B} & 0.16 & 6.0 & 1.2 \\ 48 \mathrm{R}-33 \mathrm{R} & \mathrm{Ap} & 0.34 & 14 & 6.2 \\ 45 \mathrm{R} & \mathrm{Ap} & 5.1 & 14 & 92.8 \\ 32 \mathrm{R}-27 \mathrm{R} & \mathrm{Al} & 0.04 & 4.2 & 0.2 \\ \text { 26R-23R } & \mathrm{C}-\mathrm{M} & 0.03 & 1.7 & 0.1\end{array}$

Gascoyne Abyssal Plain

$123-766 \mathrm{~A}-$

$\begin{array}{llrcr}33 \mathrm{R}-44 \mathrm{R} & \mathrm{V}-\mathrm{H} & 0.47 & 60 & 36.7 \\ 32 \mathrm{R}-25 \mathrm{R} & \mathrm{B} & 0.39 & 10 & 5.1 \\ 24 \mathrm{R}-13 \mathrm{R} & \text { Ap-Ca } & <0.01 & 3.2 & <0.1 \\ 12 \mathrm{R}-10 \mathrm{R} & \mathrm{Ca}-\mathrm{M} & <0.01 & 1.7 & <0.1\end{array}$

$\mathrm{Al}=$ Albian, $\mathrm{Ap}=$ Aptian, $\mathrm{B}=$ Barremian, $\mathrm{Be}=$ Berriasian, $\mathrm{C}=$ Cenomanian, $\mathrm{Ca}=$ Campanian, $\mathrm{M}=$ Maestrichtian, $\mathrm{H}=$ Hauterivian, $\mathrm{V}=$ Valanginian.

TOC accumulation rate $=$ average TOC $(\mathrm{wt} \%) \times$ sedimentation rate $(\mathrm{m} / \mathrm{my}) \times(1-$ porosity $) \times$ grain density $\left(\mathrm{g} / \mathrm{cm}^{3}\right)$.

The table was assembled using constant sedimentation rate intervals (Ludden. Gradstein, et al., 1990) and the average TOC (calculated from all Rock-Eval data, Ludden, Gradstein, et al., (1990)) in that interval. A porosity of $50 \%$ and a grain density of 2.6 was used for all calculations.

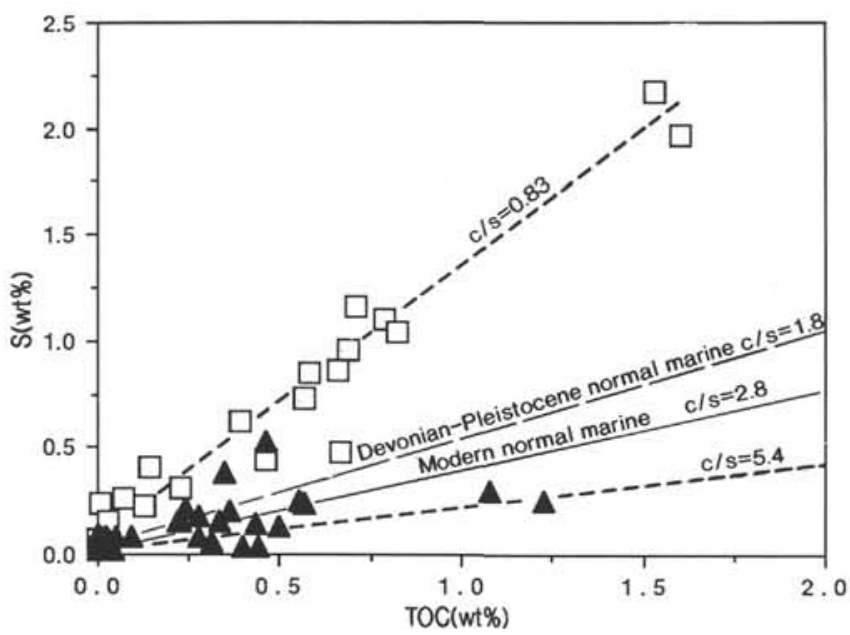

Figure 5. TOC (wt \%) and S (wt \%) from Cretaceous sediments of the Argo (Site $765=$ closed triangles) and Gascoyne (Site $766=$ open squares) abyssal plains.

sediments. Frequent occurrences of pyrite concretions and nodules were noted in the Lower Cretaceous sediments from Gascoyne Abyssal Plain, but only minor to trace amounts of pyrite were scattered throughout the Lower Cretaceous sediments from the Argo Abyssal Plain (Ludden, Gradstein, et al. 1990). Plots of pyrite sulfur vs. organic carbon, with an extension of the line of best-fit that passes through the origin, indicate deposition in normal marine sediments (Berner and Raiswell, 1983; Leventhal, 1983; Berner, 1984). The plots of Figure 5 (i.e., of TOC $<2 \mathrm{wt} \%$ ) and evidence of bioturbation at both sites (Tables 3 and 4) indicate that deposition occurred under normal marine conditions (the small positive intercept at low TOC concentrations is probably residual pore water sulfate $<0.2 \mathrm{wt} \%$ ). Deposition under euxinic conditions (anoxic water column) results in a non-zero intercept on the $\mathrm{S}$ axis (as additional pyrite can be formed in the anoxic water column) and a poor correlation between TOC and S with increased deposition of organic carbon (Leventhal, 1983; Berner and Raiswell, 1983; Berner, 1984; Raiswell and Berner, 1986). The thin $(1 \mathrm{~cm})$ interval from the Argo Abyssal Plain with a high TOC $(5.1 \mathrm{wt} \%)$ and a C/S of 0.5 may have been deposited from an anoxic water column (see below).

\section{C/S, Source, and Reactivity of Organic Matter}

The mean C/S ratio of near five from Site 765 sediments (Table 3 , Fig. 5) is higher than (1) the average of 1.2 for a variety of Cretaceous normal marine shales, (2) the average of $1.8 \pm 0.5$ quoted for Devonian through Tertiary shales (Raiswell and Berner, 1986), and (3) the modern normal marine value of 2.8 (Berner, 1982; Berner and Raiswell, 1983; Raiswell and Berner, 1986). This value near five for the Lower Cretaceous sediments results from a combination of factors. Site 765 has always been a relatively deep-water marine location with an initial water depth of $\sim 2.5 \mathrm{~km}$ (Ludden, Gradstein, et al., 1990). Hence sulfate limitation, because of low salinity (Berner and Raiswell, 1984), cannot be considered as a control on pyrite formation in these sediments. Furthermore, the sediments are primarily claystones low in calcium carbonate (Table 1) with abundant iron and iron limitation is not a constraint (see below) on pyrite formation (Berner, 1984; Raiswell and Berner, 1986).

Consequently, controls on pyrite formation are limited to the type and reactivity of organic matter accumulating in the sediments. Increasing proportions of land plant debris in sediments, i.e., increasing proportions of nonmetabolizable organic matter for the sulfate-reducing bacteria, relative to marine organic matter (Lyons and Gaudette, 1979), result in a decreasing abundance of pyrite, hence increasing $\mathrm{C} / \mathrm{S}$ ratios. The reactivity of the organic matter to the sulfate-reducing bacteria in the sediments, and hence production of $\mathrm{H}_{2} \mathrm{~S}$ for pyrite formation, is also reduced as organic matter is oxidized in the overlying water. Site 765 , with an initial water depth near $2.5 \mathrm{~km}$, rapidly subsided during the Early Cretaceous to near its present-day water depth (Ludden, Gradstein, et al., 1990). Hence, progressive oxidation of organic carbon as it settles to abyssal depths would be expected to result in increasing $\mathrm{C} / \mathrm{S}$ ratios in the sediments as an excess of refractory organic matter accumulates over pyrite produced from residual metabolizable organic matter. Extensive bioturbation of the sediments, a process that results in preferential loss of $\mathrm{H}_{2} \mathrm{~S}$ (Berner and Westrich, 1985) also contributes to increasing C/S ratios.

The good C/S correlation, with extension of the line of best-fit through the origin for Gascoyne Abyssal Plain (Site 766) sediments (Fig. 5), and a mean $\mathrm{C} / \mathrm{S}$ ratio of 0.8 , indicates carbon is not limiting in pyrite formation, pyrite is diagenetic, and deposition occurred under normal marine conditions. This value $(0.8)$ for these Lower Cretaceous sediments from the Gascoyne Abyssal Plain, is considerably lower than the mean $(C / S=5.4)$ found from Argo Abyssal Plain sediments and is indicative of the burial of organic matter that is reactive to the sulfate-reducing bacteria, from which abundant pyrite is formed. Site 766 had an inferred initial water depth around $800 \mathrm{~m}$ (Ludden, Gradstein, et al., 1990) and relatively high sedimentation rates during the Early Cretaceous (Table 5). These factors are consistent with the burial of reactive organic matter. Because pyrite sulfur was not measured directly, a component of non-pyritic sulfur in these samples could have resulted in an artificial lowering of $\mathrm{C} / \mathrm{S}$ ratios. Furthermore, the loss of organic carbon via methane production during catagenesis (moderate heating of buried organic matter) and/or bacterial activity during diagenesis contributed to the lowering of $\mathrm{C} / \mathrm{S}$ ratios in Devonian to Pleistocene sedimentary rocks in contrast to modern normal marine sediments (Raiswell and Berner, 1986). 
The sediments from this site were immature (Ludden, Gradstein, et al., 1990), excluding the possibility of production of high concentrations of thermogenic methane. However, methane and other light hydrocarbon gases were detected in these Lower Cretaceous sediments, $\sim 2-3$ times the "background" concentrations, indicating some organic carbon degradation via methanogenic microbial activity.

\section{Rock-Eval Pyrolysis and Biomarker Geochemistry}

The shipboard Rock-Eval data for the Lower Cretaceous samples of Sites 765 and 766 are shown in Figure 6. These data cluster about the Type III evolution line, indicating a low HI (hydrogen index) and organic matter depleted in hydrogen. However, pyrolysis yields and $\mathrm{HI}$ values have probably been depressed because of the mineral matrix effect associated with high clay contents in these samples (Espitalie et al., 1980; Orr, 1983; Katz, 1983; Peters, 1986).

More definitive information about organic matter source was obtained from gas chromatography and GC-MS analyses of the saturated hydrocarbon extract from selected samples (Heggie et al., this volume). The saturated hydrocarbon abundances and the ratios of $n-\mathrm{C}_{27} / n-\mathrm{C}_{17}$ were determined on eleven samples; eight from Site 766 and three from Site 765 . A variety of biomarkers from the saturates extract were measured on seven of these samples.

The presence of the $\mathrm{C}_{30}$ sterane, 24-n-propylcholestane is a specific marine biomarker (Moldowan et al., 1985, 1990), its presence and relative abundances in samples from both sites are similar to both the Mesozoic marine Toolebuc Formation in northeastern Australia (Boreham and Powell, 1987) and the marine Monterey Formation of California (Curiale and Odermatt, 1985). Furthermore, the presence of the 4-methylsterane in all samples

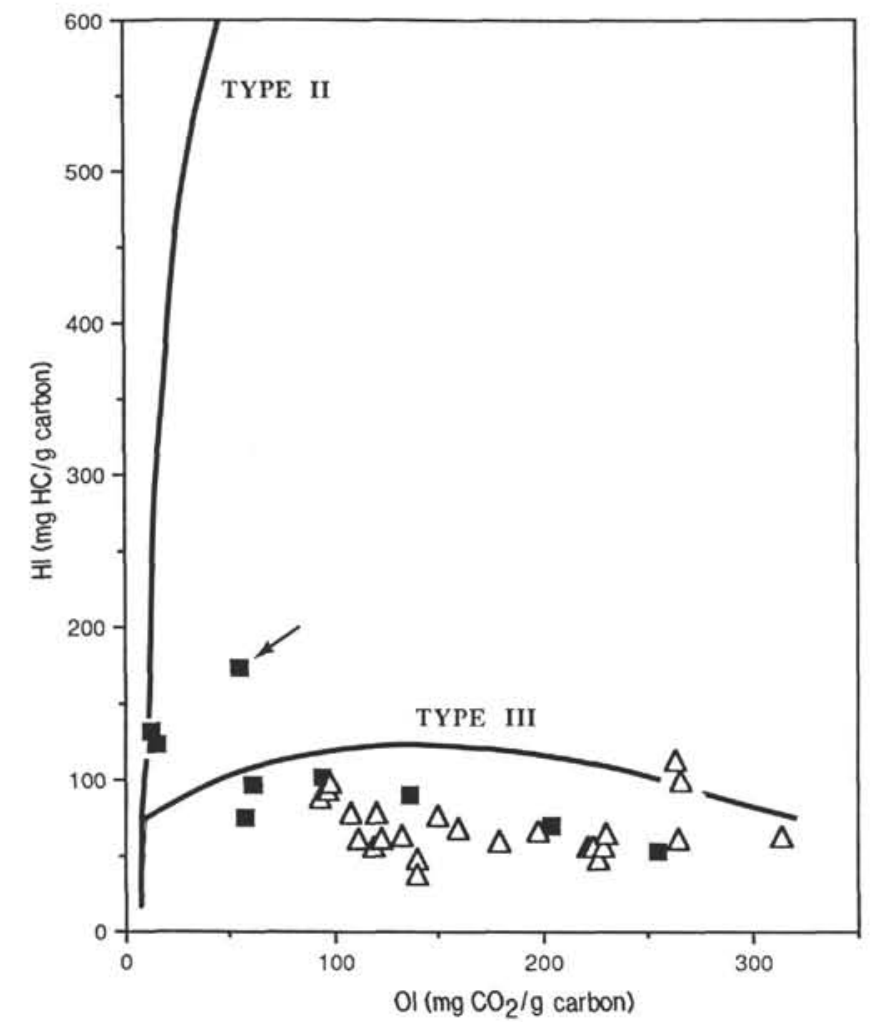

Figure 6. Rock-Eval HI vs. OI diagram of Lower Cretaceous sediments from Site 765 (closed squares) and Site 766 (open triangles). $\rightarrow$ sample with TOC $=5.1 \mathrm{wt} \%$. from both sites, with the 23,24 dimethyl side chain, is specific for and indicative of marine dinoflagellates (Summons et al., 1987). However, GC analyses of the saturated hydrocarbon extracts and the ratios of $n-\mathrm{C}_{27} / n-\mathrm{C}_{17}$ (Tissot and Welte, 1984), indicated contributions of terrestrial organic matter in two samples from the Aptian and Barremian sections of Site 765. Two samples of Barremian age, from Site 766, showed significant contributions of terrestrial organic matter, while all other GC extracts indicated a predominance of marine organic matter at Site 766. These limited data from both sites therefore indicate that the organic matter extracted from the sediments was primarily of a marine source, but contained varying contributions of terrestrial organic matter.

Shipboard palynological observations (Ludden, Gradstein, et al. 1990) indicated abundant dinoflagellate cysts in the Lower Cretaceous sediments from both sites. Spores and pollen were found scattered throughout the sediments from both sites. However, at Site 765 spores and pollen appeared more abundant in the upper Aptian to lower Albian sections than elsewhere. At Site 766 , spores and pollen were found in the $M$. australis zone (mostly of Barremian age), where they constituted $40 \%-80 \%$ of the palynomorph assemblages, although it was not possible to differentiate between Lower Cretaceous and reworked Jurassic grains (Ludden, Gradstein, et al., 1990).

Collectively, the organic geochemical analyses and the lower abundances of pyrite in sediments from the Argo Abyssal Plain, as compared to those from Gascoyne Abyssal Plain, are consistent with the burial, at Argo Abyssal Plain, of organic matter less reactive to the sulfate-reducing bacteria, through combinations of mixed terrestrial and oxidized marine sources.

\section{Carbon/Phosphorus Ratios}

The C/P ratios are calculated from the TOC and total phosphorus data and summarized in Tables 3 and 4 , and Figure 7. While the data only approximate $\mathrm{C} / \mathrm{P}$ ratios in the residual organic matter, a comparison of the ratios between the sites provides clues to the nature of the organic matter in the sediments. Most samples from the Argo Abyssal Plain (those samples with TOC $<2$ wt $\%$ ) have $C / P$ ratios $<10$. The one sample with TOC of $5.1 \mathrm{wt} \%$ has a $\mathrm{C} / \mathrm{P}$ ratio of 40 . These data contrast to those from the Gascoyne Abyssal Plain site, where the $\mathrm{C} / \mathrm{P}$ ratios are as high as 60 and more comparable to the Redfield ratio of 105 for marine organic matter. The contrast between the sites indicates that for comparable TOC

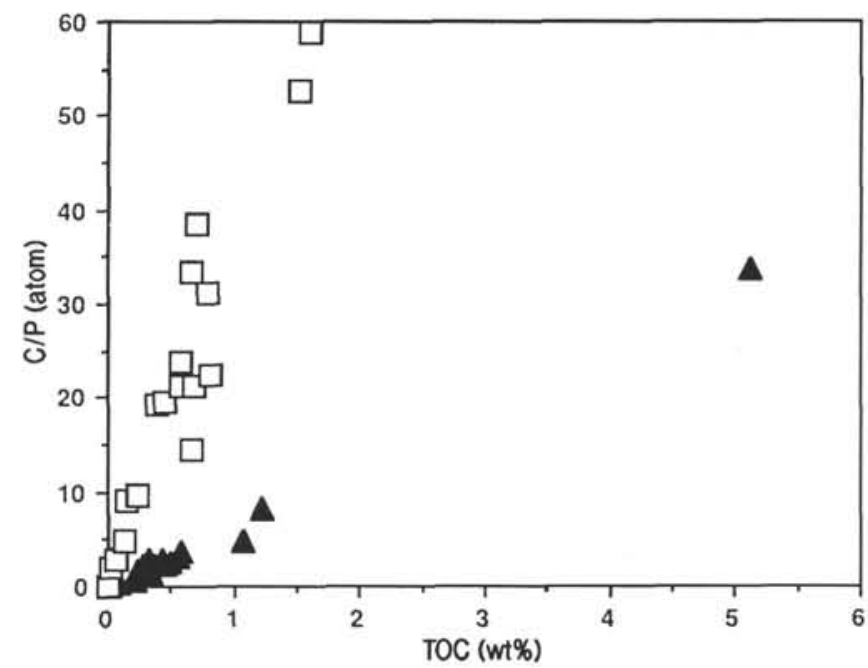

Figure 7. TOC (wt\%) and C/P (atomic) ratios in sediments for the Argo (closed triangles) and Gascoyne (open squares) abyssal plains. 
values, the deeper-water site of the Argo Abyssal Plain contains more residual $P$. These data and the trend of Figure 7 suggest preferential remobilization of organic carbon (over phosphorus) with increasing water depth. The result is compatible with observations summarized in Ingall and Van Cappellen (1990) of low $\mathrm{C} / \mathrm{P}$ ratios in organic matter in pelagic sediments. The prolonged degradation of organic carbon, such as occurs in pelagic sediments, apparently leads to the formation of phosphorus-enriched organic matter (Froelich, 1982).

\section{Ferrous and Pyrite Iron}

Ferrous iron is produced as a byproduct of organic matter oxidation in sediments under sub-oxic conditions (e.g., Froelich et al., 1979) following the depletion of pore-water oxygen and nitrate, but prior to the onset of bacterial sulfate reduction. Sedimentary iron oxyhydroxide reduction and the release of ferrous iron to pore waters has been documented in pelagic and hemipelagic sediments (e.g., Klinkhammer, 1980; Bender and Heggie, 1984; Heggie et al., 1987). Ferrous iron, once released to pore waters, may be redeposited in authigenic minerals or, where sufficient reactive organic carbon is buried in the sediments to deplete the primary oxidants, reacts with hydrogen sulfide (produced during bacterial sulfate reduction of sedimentary organic matter) to form iron monosulfides and ultimately pyrite (e.g., Froelich et al., 1979; Berner, 1981; Berner, 1982; Curtis, 1985).

Sedimentary ferrous iron abundances have been investigated as empirical indicators of sub-oxic conditions for the following reasons: (1) frequent appearances of red sediments, the color banding and gradations from red through gray, green to black, and (2) the fact that pyrite was observed scattered in only minor to trace quantities throughout these relatively deep $(>2.5 \mathrm{~km})$ Lower Cretaceous sediments of Site 765. Ferrous iron (although in unidentified minerals) but no pyrite, suggests sub-oxic conditions (implying relatively low organic carbon burial fluxes and/or a plentiful supply of the primary oxidants, oxygen and nitrate), while pyrite occurrences are indicative of anoxic conditions, sulfate reduction, and higher organic carbon burial fluxes. Ferrous and pyrite iron abundances, when considered together, may be empirical indicators of fluctuating sub-oxic and anoxic conditions.

Different modes of pyrite formation, including diagenetic, syngenetic (formed before burial both in the water column and at the sediment/seawater interface), iron-limited and carbon-limited under euxinic (anoxic, $\mathrm{H}_{2} \mathrm{~S}$ bottom waters) and near-euxinic (very low oxygen bottom waters) conditions, have been distinguished by the degree of pyritization (DOP) (Raiswell and Berner, 1985). DOP, after Berner (1970), is defined as pyrite iron/total iron, although the sum of pyrite iron plus iron soluble in hot concentrated hydrochloric acid (reactive iron) is a better indicator of the iron (rather than total iron) that is available to hydrogen sulfide to form pyrite. However, in practice both total iron and reactive iron have been used in this application of DOP (Raiswell and Berner, 1985; Donnelly et al., 1988). DOP values calculated here are subject to two approximations. First, pyrite iron has been calculated stoichiometrically from the total sulfur content, assuming (discussed earlier) that all sulfur occurs as pyrite. Second, total rather than reactive iron has been used in the denominator of DOP. The data of Raiswell and Biatty (in press) show, for a variety of Devonian-Cretaceous, Cambrian-Silurian, and modern sediments of the Amazon shelf, that $\mathrm{HCl}$-soluble iron and total iron are linearly related, although the proportion of $\mathrm{HCl}$-soluble to total iron may vary for sediments of different ages. Donnelly et al. (1988), found that $\mathrm{HCl}$-soluble iron was $~ 30 \%$ of total iron in samples of middle Cambrian age. Because reactive iron for pyrite formation is always less than total iron, the DOP values calculated here underestimate those calculated if the sum of pyrite iron and $\mathrm{HCl}$-soluble iron had been used in the denominator of DOP.

\section{Total Organic Carbon (TOC) and Degree of Pyritization (DOP)}

DOP values are plotted vs. TOC in Figure $8 \mathrm{~A}$. The data from Site 766 are correlated $(R=0.90)$, while that from Site 765 are poorly correlated $(\mathrm{R}=0.55)$. For $\mathrm{TOC}<2 \mathrm{wt} \%$, the correlations indicate iron is available for pyrite formation, pyrite is diagenetic and, as the data pass through the origin, indicate deposition has occurred in normal marine sediments. A consideration affecting the relation between pyrite sulfur and organic carbon is whether or not the original deposition of organic matter was coupled to the deposition of iron through the association of colloidal organic matter and iron oxide particles with fine clay grains (Berner, 1984; Raiswell and Berner, 1985). Total iron (used here as a proxy for reactive iron) does not correlate with TOC in the sediments from either the Argo or Gascoyne abyssal plains (Fig. 8B). Hence, pyrite iron formation is diagenetic (Fig. 8A) and not an artifact,
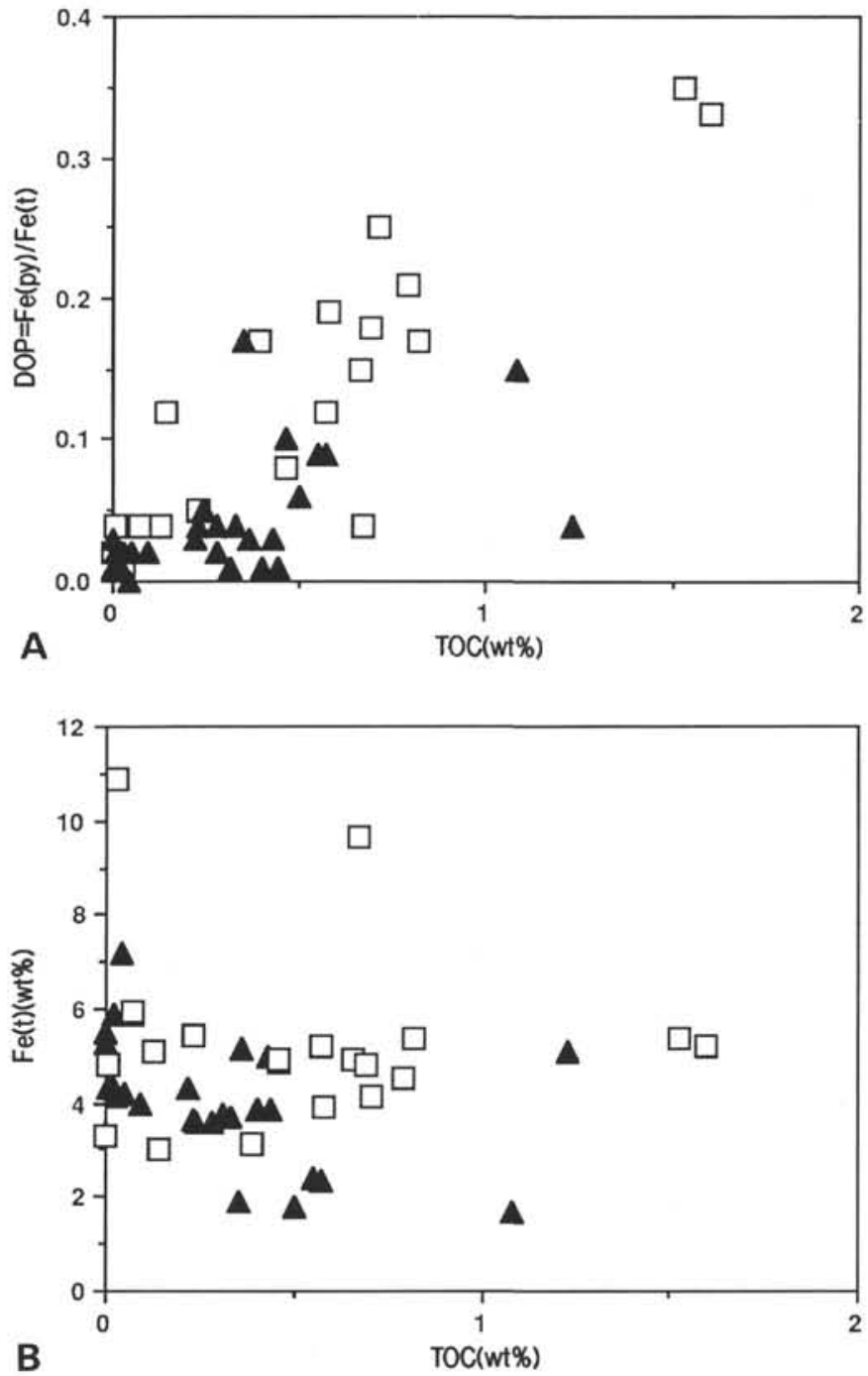

Figure 8. A. TOC and DOP (degree of pyritization) for Argo (closed triangles) and Gascoyne (open squares) Abyssal Plain sediments. B. TOC and total iron, $\mathrm{Fe}(\mathrm{t})$, for Argo (closed triangles) and Gascoyne (open squares) Abyssal Plain sediments. 
because organic carbon and iron are not associated in the original depositing materials.

Raiswell et al. (1988) proposed that the degree of pyritization (DOP) could be used as an indicator of bottom-water oxygen conditions. These authors examined sediments of various ages classified on paleoecological and sedimentological criteria as being deposited in aerobic ( $\mathrm{DOP}<0.42)$, restricted $(0.46<\mathrm{DOP}<$ $0.8)$, or inhospitable (anoxic) bottom waters $(0.55<\mathrm{DOP}<0.93)$. Because of the approximations noted above, the DOP values here are not directly comparable to those of Raiswell et al. (1988).

For TOC $<2 \mathrm{wt} \%$, all DOP values are $\leq 0.4$ (Tables 3 and 4 ). The TOC-rich sample from the Argo Abyssal Plain, sampled near the Barremian-Aptian boundary, had a DOP value of 0.9. The approximations, noted above, of calculated DOP values, combined with the considerable overlap in DOP between restricted and inhospitable (anoxic) conditions (Raiswell et al., 1988), indicate the interpretation of a DOP of 0.9 (deposition from an anoxic water column) is equivocal. However, other observations (Thurow, pers. comm., 1991) support an interpretation of anoxic bottom water conditions. This sample had the following characteristics: (1) under a scanning electron microscope, it was almost entirely framboidal pyrite; (2) this thin interval was finely laminated with no evidence of bioturbation; and (3) it had clay mineralogy similar to that from adjacent samples, suggesting it was not derived from another sediment provenance. Finally, in Figure 9, total iron vs. total $\mathrm{Al}_{2} \mathrm{O}_{3}$ is plotted for both Gascoyne and Argo abyssal plains samples. If all iron in the sediments were detrital, values would be expected to cluster about the average $\mathrm{Fe}(\mathrm{t}) / \mathrm{Al}_{2} \mathrm{O}_{3}$ ratio as shown by the solid line. The excess iron indicated on Figure 9, for the TOC-rich sample may be pyrite iron that is formed by precipitation in an ancxic water column, settles to the sediment surface, and is subsequently incorporated into the sediments. Therefore, this TOC-rich sample may be either the local expression of the broadly defined Barremian-Albian Oceanic Anoxic Event (OAE-1) of Schlanger and Jenkyns (1976) or a sub-event of OAE-1. This interpretation will require more detailed analyses to confirm, but there was insufficient sample available from this interval for further work. Such a high abundance of pyrite in this sample suggests deposition of organic matter reactive to the sulfate-reducing bacteria. However, RockEval pyrolysis of this and adjacent samples (Fig. 6) did not provide any clues to changes in the composition of the organic matter being deposited.

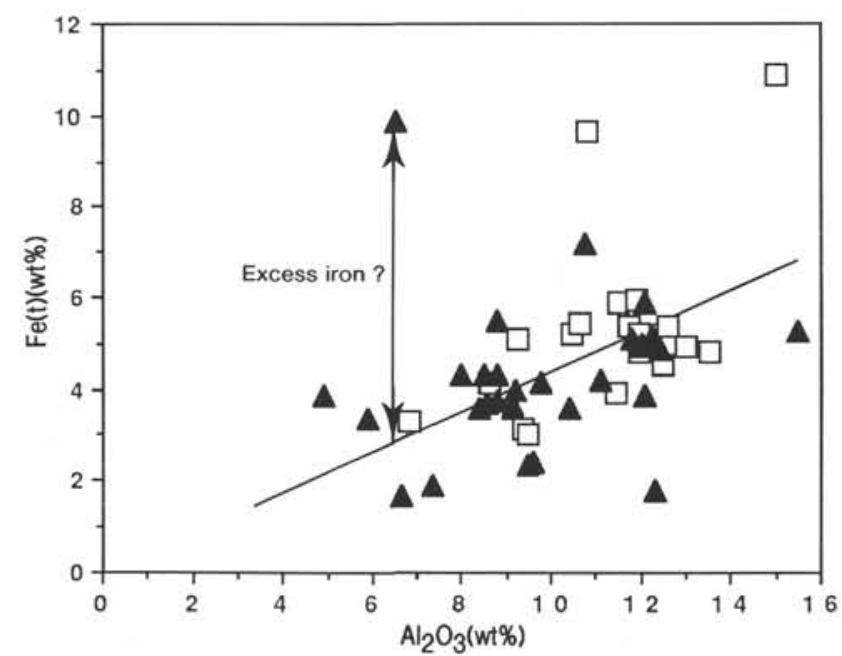

Figure 9. Total iron $\mathrm{Fe}(\mathrm{t})$ and $\mathrm{Al}_{2} \mathrm{O}_{3}$ (wt\%) for the $\mathrm{Argo}$ (closed triangles) and Gascoyne (open squares) abyssal plains, Lower Cretaceous sediments.

\section{Ferrous and Pyrite Iron and Color-Banded Sediments}

Plots of ferrous iron/total iron and ferrous iron + pyrite iron/total iron are summarized for both locations in Figure 10. Ferrous iron abundances increase with increasing TOC at both sites (Figs. 10A and 10B) and indicate that ferrous iron is diagenetic. However, at low TOC $(<0.2 \mathrm{wt} \%)$ at Site $766, \mathrm{FeII} / \mathrm{Fe}(\mathrm{t})$ was high in two samples and the data trend to intercept the $\mathrm{Fe}$ speciation axis at a non-zero value (Fig. 10A), which suggests a non-diagenetic or detrital ferrous iron component.

Ferrous iron abundances (for TOC $<2 \mathrm{wt} \%$ ) are significant in these sediments and represent $<30 \%$ and $<40 \%$ of total iron at the Argo and Gascoyne abyssal plains, respectively. However, in the sample of high TOC (5 wt\%), from near the Barremian/Aptian boundary, $\mathrm{FeII} / \mathrm{Fe}(\mathrm{t})$ is $<0.2$, and most iron $(>80 \%)$ occurs as pyrite (Fig. 10C). These data suggest sedimentary ferrous iron increases with increases in TOC $(\leq \sim 2 \mathrm{wt} \%)$, which is ultimately depleted as additional TOC is buried and pyrite begins to form to become the ultimate sink for remobilized iron. The numerical differences on the Fe speciation axes between the (ferrous iron + pyrite iron) and ferrous iron represent DOP values discussed in Figure $8 \mathrm{~A}$. The sum of ferrous plus pyrite iron (for TOC $<2 \mathrm{wt} \%$ ) represents $\leq \sim 70 \%$ of total iron at Gascoyne Abyssal Plain and $50 \%$ at Argo Abyssal Plain (Figs. 10A and 10B).

At Argo Abyssal Plain, the distributions of sedimentary ferrous iron (Table 3 ), indicate: (1) the highest abundances of ferrous iron ( $\leq 27 \%$ of total iron) in the dark-green colored Aptian claystones of Unit IVC (TOC $<0.5 \mathrm{wt} \%$ ); (2) that ferrous iron is typically $10 \%-20 \%$ of total iron in the gray and green claystones of Units VB and VC (TOC 0.1-1.2 wt\%); and (3) the lowest abundances of ferrous iron ( $<10 \%$ of total iron) are found in those red colored claystones predominant in Units VC, VI, and VIl (TOC $<0.2 \mathrm{wt} \%$, Table 3). Pyrite iron abundances show a similar trend, being highest in samples with high TOC and the dark-gray and green Barremian and Aptian samples, and lowest in the red claystones of Berriasian through Valanginian ages.

Selected samples of the color-banded (red, dark-green, darkgray, black) claystones from the Lower Cretaceous sediment Units VA-VII, were analyzed by X-ray diffraction. The results are summarized in Table 6 and indicate that all red samples consistently contained oxidized iron minerals, i.e., hematite and/or goethite, and these were absent in the light gray and gray-green samples. Pyrite (with a detection limit of $\sim 5 \mathrm{wt} \%$ ) was not detected in any samples. Red-colored samples always have the lowest abundances of both pyrite and ferrous iron (Table 3).

\section{SUMMARY}

The abundances of some diagenetic byproducts that resulted from the burial, degradation, and preservation of organic matter were compared in samples of Lower Cretaceous sediments from the Argo and Gascoyne abyssal plains.

1. Both pyrite (estimated from total sulfur) and TOC are generally more abundant in the Gascoyne Abyssal Plain than the Argo Abyssal Plain, although the sample of highest TOC was measured in a thin $(1 \mathrm{~cm})$ layer from the Argo Abyssal Plain near the Aptian/Barremian boundary.

2 . The average $\mathrm{C} / \mathrm{S}$ ratio (for samples of TOC $<2 \mathrm{wt} \%$ ) in Argo Abyssal Plain sediments is $\sim 5$. This result reflects the burial of an excess of organic carbon that was unreactive to the sulfate-reducing bacteria, over pyrite formed from metabolizable organic carbon during sulfate reduction. The $\mathrm{C} / \mathrm{S}$ ratio in the high TOC sample from the Aptian is 0.5 and suggests deposition of a source of organic matter that was reactive to the sulfate-reducing bacteria. In contrast to the data from the Argo Abyssal Plain (TOC $<2 \mathrm{wt} \%$ ), the average $\mathrm{C} / \mathrm{S}$ ratio from the Gascoyne Abyssal Plain sediments 


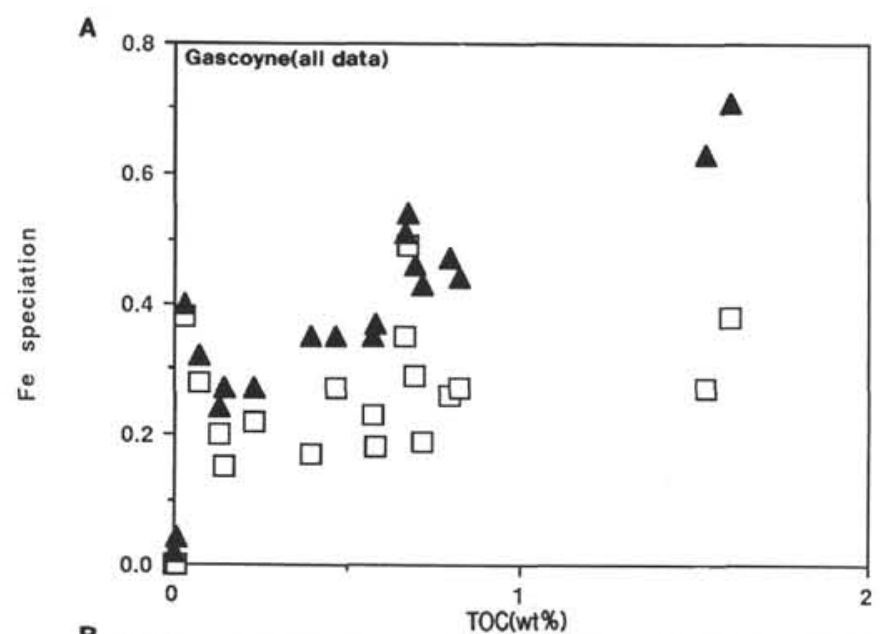

Table 6. XRD analyses of select color-banded Aptian-Hauterivian sediments from the Argo Abyssal Plain.

\begin{tabular}{|c|c|c|c|}
\hline $\begin{array}{l}\text { Core, section, } \\
\text { interval }(\mathrm{cm})\end{array}$ & $\begin{array}{l}\text { Depth } \\
\text { (mbsf) }\end{array}$ & Color & Description \\
\hline \multicolumn{4}{|l|}{$123-765 \mathrm{C}-$} \\
\hline $40 \mathrm{R}-4,143-144$ & 726.83 & gray & $\begin{array}{l}\text { Quartz minor, feldspar trace, mont- } \\
\text { morillonite major, mica trace }\end{array}$ \\
\hline $47 R-3,55-57$ & 790.95 & It red & $\begin{array}{l}\text { Quartz major, hematite trace, feld- } \\
\text { spar trace, montmorillonite } \\
\text { major, mica trace }\end{array}$ \\
\hline $50 \mathrm{R}-2,80-82$ & 817.90 & gray/green & $\begin{array}{l}\text { Quartz major, feldspar trace, mont- } \\
\text { morillonite major, mica trace, } \\
\text { chlorite trace }\end{array}$ \\
\hline $52 \mathrm{R}-1,132-133$ & 835.82 & It gray & $\begin{array}{l}\text { Quartz major, feldspar trace, mont- } \\
\text { morillonite minor mica trace }\end{array}$ \\
\hline $52 \mathrm{R}-2,8-10$ & 836.08 & It gray & $\begin{array}{l}\text { Quartz major, montmorillonite } \\
\text { minor, mica trace }\end{array}$ \\
\hline $52 \mathrm{R}-2,10-11$ & 836.10 & gray/green & $\begin{array}{l}\text { Quartz major, feldspar trace, mont- } \\
\text { morillonite minor, kaolinite } \\
\text { trace }\end{array}$ \\
\hline $53 \mathrm{R}-3,72-74$ & 847.82 & It red & $\begin{array}{l}\text { Quartz major, feldspar trace, hema- } \\
\text { tite trace, montmorillonite } \\
\text { minor, mica trace }\end{array}$ \\
\hline $55 \mathrm{R}-2,82-84$ & 865.32 & It red & $\begin{array}{l}\text { Quartz major, hematite trace, mont- } \\
\text { morillonite minor, mica trace }\end{array}$ \\
\hline $57 \mathrm{R}-7,15-17$ & 890.55 & It red & $\begin{array}{l}\text { Quartz major, hematite trace, mont- } \\
\text { morillonite trace, mica trace }\end{array}$ \\
\hline $59 \mathrm{R}-4,111-112$ & 903.50 & It gray & $\begin{array}{l}\text { Quartz major, feldspar trace, mont- } \\
\text { morillonite trace, mica trace }\end{array}$ \\
\hline $60 R-2,61-63$ & 909.61 & dark red & $\begin{array}{l}\text { Quartz major, feldspar trace, hema- } \\
\text { tite trace, montmorillonite } \\
\text { minor, mica minor }\end{array}$ \\
\hline $61 \mathrm{R}-5,105-106$ & 923.95 & $\mathrm{red} /$ brown & $\begin{array}{l}\text { Calcite major, quartz major, feld- } \\
\text { spar trace, goethite trace, } \\
\text { montmorillonite minor, mica } \\
\text { trace }\end{array}$ \\
\hline
\end{tabular}

is 0.8 and indicates burial of organic matter from which abundant pyrite was formed.

3. The organic carbon/total phosphorus ratios in the sediments are low $(<10)$ in the Argo Abyssal Plain compared to those in the Gascoyne Abyssal Plain ( 60) for comparable TOC contents. These ratios are lower than the Redfield ratio of 105 for marine organic matter and suggest preferential remobilization of organic carbon over phosphorus with increasing water depth.

4. A consideration of the iron mass balance, including the amounts of ferrous iron and pyrite iron (expressed as a fraction of total iron in the sediments), indicate generally higher abundances of both ferrous and pyrite iron at the Gascoyne Abyssal Plain site (for TOC $<2 \mathrm{wt} \%$ ). Ferrous iron and pyrite iron are related to TOC (although poorly correlated in the Argo Abyssal Plain samples) in the sediments from both sites and therefore are diagenetic byproducts. The estimates of DOP (the degree of pyritization) and evidence of bioturbation support the results of the TOC vs. S plots, that for TOC $<2 \mathrm{wt} \%$ deposition has occurred in normal marine sediments. One sample, near the Barremian/Aptian boundary, with $5.1 \mathrm{wt} \%$ TOC, abundant pyrite, fine laminations, and no bioturbation may represent deposition during a brief period of anoxia in the overlying water, thus representing the local expression of the Barremian-Albian Oceanic Anoxic Event.

\section{ACKNOWLEDGMENTS}

Figure 10. A. TOC and ferrous iron/total iron (FeII/Fe[t], open squares) and ferrous iron + pyrite iron/total iron (Fell + $\mathrm{Fe}[\mathrm{py})] / \mathrm{Fe}[\mathrm{t}]$, closed triangles) for the Gascoyne Abyssal Plain sediments (TOC $<2$ wt\%). B. TOC and ferrous iron/total iron (FeII/Fe[t], open squares) and ferrous iron + pyrite iron (FeII + $\mathrm{Fe}[\mathrm{py}] / \mathrm{Fe}[\mathrm{t}]$, closed triangles) for Argo Abyssal Plain sediments (TOC $<2 \mathrm{wt} \%$ ). C. TOC and ferrous iron/total iron (FeII/Fe[t], open squares) and ferrous iron + pyrite iron/total iron (FeII + Fe[py]/Fe[t], closed triangles) for Argo Abyssal Plain sediments (all data).

The XRF and FeO analyses were conducted at the Division of Onshore Sedimentary and Petroleum Geology, Bureau of Mineral Resources, under the supervision of John Pyke and Bruce Cruikshank. Julie Kamprad did the XRD analyses. I thank T. Donnelly for several helpful discussions and his critical and constructive comments on an earlier version of this manuscript. G. Hieshema, T. Powell, and C. Boreham also provided constructive comments 
on an earlier manuscript. K. H. Wolf, BMR editor, assisted in the preparation of the final manuscript. DTH publishes with the permission of the Executive Director of the Bureau of Mineral Resources, Canberra, Australia.

\section{REFERENCES}

Bender, M. L., and Heggie, D. T., 1984. The fate of organic carbon on the sea-floor: a status report. Geochim. Cosmochim. Acta., 48: 977986.

Berner, R. A., 1970. Sedimentary pyrite formation. Am. J. Sci., 268:1-23. 1981. Authigenic mineral formation resulting from organic matter decomposition in modern sediments. Fortschr. Miner., 59: $117-135$.

1982. Burial of organic carbon and pyrite sulfur in the modern ocean: its geochemical and environmental significance. Am. J. Sci., 282:451-473.

1984. Sedimentary pyrite formation: an update. Geochim. Cosmochim. Acta, 48:605-615.

Berner, R. A., and Raiswell, R., 1983. Burial of organic carbon and pyrite sulfur in sediments over Phanerozoic time: a new theory. Geochim. Cosmochim. Acta., 47:855-862.

, 1984. C/S method for distinguishing freshwater from marine sedimentary rocks. Geology, 12:365-368.

Berner, R. A., and Westrich, J. T., 1985. Bioturbation and the early diagenesis of carbon and sulfur. Am. J. Sci., 285:193-206.

Boreham, C. J., and Powell, T. G. ,1987. Sources and preservation of organic matter in the Toolebuc Formation, eastern Australia. Org. Geochem., 11:433-449.

Curiale, J. A., and Odermatt, J. R., 1989. Short-term biomarker variability in the Monterey Formation, Santa Maria Basin. Org. Geochem, 14:113.

Curtis, C. D., 1985. Clay mineral precipitation and transformation during burial diagenesis. Phil. Trans, R. Soc. London, A315:91-105.

Donnelly, T. H., and Crick, I. H., 1988. Depositional environment of the Middle Proterozoic Velkerri Formation in northern Australia: geochemical evidence. Precambrian Res., 42:165-172.

Donnelly, T. H., Shergold, J. H., and Southgate, P. N., 1988. Pyrite and organic matter in normal marine sediments of middle Cambrian age, southern Georgina Basin, Australia. Geochim. Cosmochim. Acta., $52: 259-263$.

Emeis, K., and Kvenvolden, K. A., 1986. Shipboard organic geochemistry on JOIDES Resolution, ODP Tech. Note 7.

Espitalié, J., Madec, M., and Tissot, B., 1980. Role of mineral matrix in kerogen pyrolysis: influence on petroleum generation and migration. AAPG Bull., 64:59-66.

Froelich, P. N., Klinkhammer, G. P, Bender, M. L., Luedtke, N. A., Heath, G. R., Cullen, D., Dauphin, P., Hammond, D., and Hartman, B., 1979. Early oxidation of organic matter in pelagic sediments of the eastern equatorial Atlantic: suboxic diagenesis. Geochim. Cosmochim. Acta., 43:1075-1090.

Froelich, P. N., Bender, M. L, Luedtke, N. A., Heath, G. R., and DeVries, T., 1982. The marine phosphorus cycle. Am. J. Sci., 28:474-511.

Heggie, D. T., Maris, C., Hudson, A., Dymond, J., Beach, R., and Cullen, J. D., 1987. Organic carbon oxidation and preservation in NW Atlantic continental margin sediments. In Weaver, P.P.E., and Thomson, J. (Eds.), Geology and Geochemistry of Abyssal Plains. Geol. Soc. Spec. Publ., 31:215-236.

Ingall, E. D., and Van Cappellen, P., 1990. Relation between sedimentation rate and burial of organic phosphorus and organic carbon in marine sediments. Geochim. Cosmochim. Acta., 54:373-386
Katz, B. J., 1983. Limitations of "Rock-Eval" pyrolysis for typing organic matter. Org. Geochem., 4:195-199.

Klinkhammer, G. P., 1980. Early diagenesis in sediments from the Eastern Equatorial Pacific, II. Pore water metal results. Earth Planet. Sci. Lett., 49:81-101.

Leventhal, J. S., 1983. An interpretation of carbon and sulfur relationships in Black Sea sediments as indicators of environments of deposition. Geochim. Cosmochim. Acta., 47:133-138.

Ludden, J. N., Gradstein, F. M., et al., 1990. Proc. ODP, Init. Repts., 123: College Station, TX (Ocean Drilling Program).

Lyons, W. B., and Gaudette, M. E., 1979. Sulfate reduction and the nature of organic matter in estuarine sediments. Org. Geochem., 1:151-155.

Moldowan, J. M., Seifert, W. K., and Gallegos, E. J., 1985. Relationship between petroleum composition and depositional environment of petroleum source rocks. AAPG Bull., 69:1255-1268.

Moldowan, J. M., Fago, F. J., Lee, C. Y., Jacobson S. R., Watt, D. S, Nacer-Eddine, S., Jeganathan, A., and Young D. C., 1990. Sedimentary 24-n-propylcholestanes, molecular fossils diagnostic of marine algae. Science, 247:309-312.

Norrish, K., and Hutton J. T, 1969. An accurate X-ray spectrographic method for the analysis of a wide range of geological samples. Geochim. Cosmochim. Acta., 33:431-453.

Peters, K. E., 1986. Guidelines for evaluating petroleum source rocks using programmed pyrolysis. AAPG Bull., 32:318-329.

Raiswell, A., and A-Biatty, in press. Depositional and diagenetic C-S-Fe signatures and their influence on metallogenetic potential. In Schidlowski, M. (Ed.), Implications for mineral and energy resources: Berlin (Springer-Verlag).

Raiswell, R., and Berner, R. A., 1985. Pyrite formation in euxinic and semi-euxinic sediments. Am. J. Sci., 285:710-724.

1986. Pyrite and organic matter in Phanerozoic normal marine shales. Geochim. Cosmochim. Acta., 50:1967-1976.

Raiswell, R., Buckley, F., Berner, R. A., and Anderson, T.F., 1988. Degree of pyritization of iron as a paleoenvironmental indicator of bottom-water oxygenation. J. Sediment. Petrol., 58:812-819.

Saxby, J. D., 1986. Geochemistry of oil shale in the eastern Eromanga Basin. In Gravestock, D. I., Moore, P. S., and Pitt, G. (Eds.), Contributions to the Geology and Hydrocarbon Potential of the Eromanga Basin. Geol. Soc. Australia Spec. Publ., 12:241-254.

Schapiro, L., and Brannock, W. W., 1961. Rapid analysis of silicate carbonate and phosphate rocks. USGS Bull., 1144A.

Schlanger, S. O., and Jenkyns, H. C., 1976. Cretaceous oceanic anoxic events: Causes and consequences. Geol. en Minj., 55:179-184.

Summon, R. E., and Powell, T. G., 1987. Identification of aryl isoprenoids in source rocks and crude oils: Biological markers for the green sulfur bacteria. Geochim. Cosmochim. Acta., 51:557-566.

Tissot, B. P., and Welte, D. H., 1984. Petroleum Formation and Occurrence: New York (Springer-Verlag).

von Rad, U., Thurow, J., Haq., B. U., Gradstein, F. M., Ludden, J., and ODP Leg 122/123 shipboard scientific parties, 1989. Triassic to Cenozoic evolution of the NW Australian continental margin and the birth of the Indian Ocean (preliminary results of ODP Legs 122 and 123). Geol. Rundschau., 78:1189-1210.

Date of initial receipt: 31 August 1990

Date of acceptance: 22 May 1991

Ms 123B-169 